\title{
A Rotation-Free Beam Element for Beam and Cable Analyses
}

\author{
Y.X.Zhou, K.Y.Sze \\ Department of Mechanical Engineering, The University of Hong Kong, Pokfulam, Hong Kong. \\ * Correspondence author (Email: kysze@hku.hk)
}

\begin{abstract}
The rotation-free or RF element method represents a non-conventional finite element method in which the rotations are not used as dofs and the element interpolation domains are overlapping. Its obvious advantage is that the complication of finite rotations can be avoided. In this paper, the relatively unexplored RF plane beam element recently formulated by the authors in the course of developing a RF triangle for thin-shell analyses is revisited. Comparing with other RF plane beam elements, the present one is simple and physical yet its accuracy remains competitive. Using a corotational approach and the small strain assumption, its tangent bending stiffness matrix can be approximated by a constant matrix which does not require updating in geometric nonlinear analyses. The element is here extended to spatial cable analyses in which the torsional stiffness can often be neglected and the sectional properties are isotropic. Under the same nodal distributions, it is seen that the present element can tolerate much larger load increment and time step under static and dynamic analyses, respectively, than the two-node thin beam finite element.
\end{abstract}

Keywords: rotation- free; beam; finite element; corotational; geometric nonlinear; cable. 


\section{INTRODUCTION}

Rotation-free or RF element method has attracted considerable attention in the last two decades. An obvious advantage of the method is that it simplifies the kinematic description under finite rotations. While the focus of the method is on RF triangular plate/shell elements [1-7], the RF beam elements are relatively unexplored until more recently. In the RF element, its interpolation domain is larger than its integration domain which is referred to as "element" for simplicity. In other words, not only the nodes within but also adjacent to the element are employed in the displacement interpolation. Phaal \& Calladina [1] developed a RF beam element based on the quadratic interpolation. Three nodes are used to construct the displacement from which a constant curvature can be derived, see Figure 1(a). To the best knowledge of the authors, this straight forward linear straight beam formulation was not extended to curved beam and nonlinear analyses. Flores \& Oñate [8] presented RF elements for nonlinear analyses of plane beams and axisymmetric shells with special emphasis on treating non-smooth and branching beams. With respect to Figure 1(b), 1-2, 2-3 and 3-4 are treated as straight and their directors $\left(\mathbf{n}_{12}, \mathbf{n}_{23}\right.$ and $\left.\mathbf{n}_{34}\right)$ after deformation are computed accordingly in Reference [8]. Based on the displaced directors, the curvatures at nodes 2 and 3 are determined and linearly interpolated for the element bounded by the two nodes. Oñate \& Zarate [9] later included the transverse shear deformation into the formulation by introducing shear angle dofs. On the other hand, Battini [10] proposed a RF co-rotational plane beam element. The element again relies on four nodes and the co-rotational frame is aligned with nodes 2 and 3. Using nodes 1 to 4 and nodes 1 to 3, cubic and quadratic local deflections are interpolated, respectively. The local rotation at node 2 is taken as the average rotations derived from the two deflections. Similarly, the local rotation at node 3 can be derived. Using the local rotations and the zero local deflections at the two nodes, another local cubic transverse deflection is derived for the element bounded by nodes 2 and 3.

Very recently, the authors have formulated a RF plane beam element in the course of developing a RF triangle plate/shell element [11]. The formulation can be regarded as an extension of the simple RF beam element of Phaal \& Calladina [1] to the curved beam and geometric nonlinear analyses. Comparing with other RF beam elements, the present one is simple and physical but its accuracy is competitive. Using a corotational approach and the small strain assumption, its tangent bending stiffness matrix can be approximated as a constant matrix which does not require updating in a geometrically nonlinear analysis. It is particularly suitable for efficient analysis of highly geometrically nonlinear problems. Cables, which are used in cable-supported bridges and roofs, are typical examples [12]. 


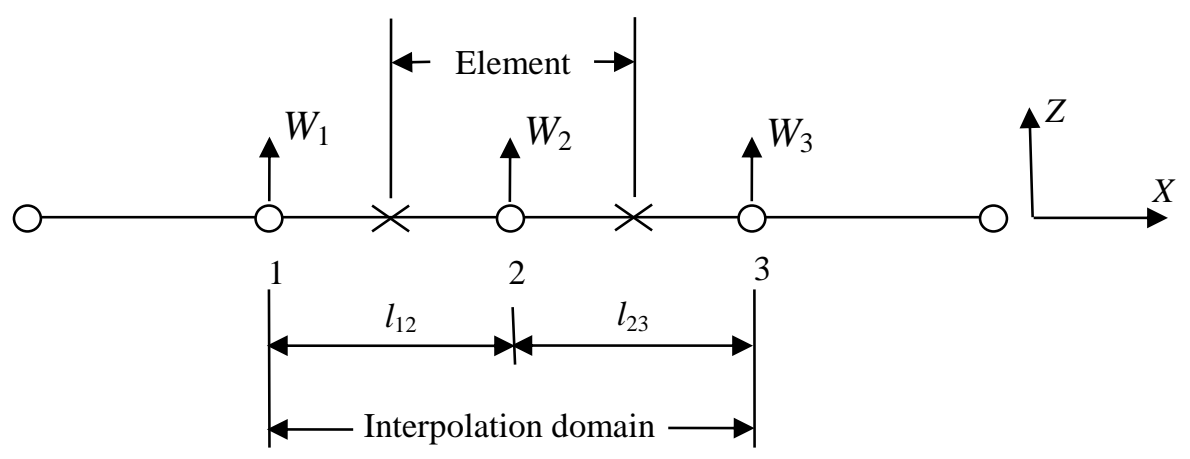

(a)

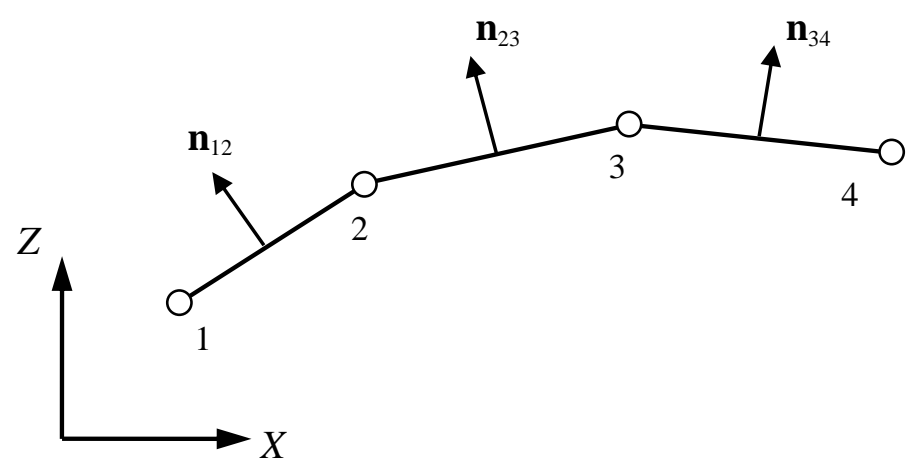

(b)

Figure 1. (a) Three consecutive nodes along a straight plane beam. (b) Four consecutive nodes along a curved plane beam.

In computational analysis of cables, linear and higher order line finite elements [13, 14], catenary finite elements $[12,15-18]$ and, of course, beam finite elements can be employed. As the catenary elements combine the analytical catenary expressions with the numerical method, they can yield accurate static predictions by using very few elements.

Nevertheless, there are a couple of drawbacks in the line and catenary elements. Firstly, they concern only the axial force and the bending effect is ignored. This is justifiable in most but not all cases. For example, Irvin [19] pointed out that when rapid changes in curvature are unavoidable, the bending effect may be locally important. Recently, Buckham et al [20] also indicated that the bending effect is sometimes important, e.g. in the dynamic simulation of slack tethers used in underwater remotely operated vehicles. Secondly, numerical instability and convergence difficulties are sometimes encountered. Thus, some additional schemes such as pre-stress, pre-strain, assumed configuration and form-finding have been proposed [21]. Nevertheless, proper choice of these schemes and the related settings are not straight forward. Of course, these drawbacks do not exist in the beam finite element. Recently, a 'nodal coordinate element' [22, 23] has been proposed to deal with aforementioned drawbacks. The element takes bending and transverse shear effects into 
account. However, in their formulation, not only the nodal coordinates but also the slopes of the coordinates need to be taken as the nodal dofs. For 3D analysis, each node carries 12 dofs. The formulation is complicated and the computational effort is considerably large.

In the present paper, the RF beam element proposed in Reference [11] will be re-visited and applied to the cable applications. The outline of this paper is as follows. The linear formulation of the beam element is reviewed in Section 2 followed by some numerical examples in Section 3. In Section 4, the corotational approach is employed to extend linear element to geometrically nonlinear analyses. Nonlinear numerical examples are given in Section 5. It should be remarked that the materials presented in Sections 2 and 4 have been similarly presented in Reference [11]. However, Reference [11] covers linear and nonlinear straight beams, curved beams, plates and shells. Consequently, only two smooth and relatively unconstrained beam examples are presented. The examples presented here are markedly different in nature from the two in Reference [11]. They include constrained and folded beams which have also been considered by other RF beams. Our RB beam is indeed comparable to those published by the others in accuracy yet its formulation is much simpler. In Section 6, a pseudo 3D RF beam element is newly developed and employed in cable analyses as presented in Section 7. Under the same nodal distributions, it can be seen that the present element (with only translational dofs) can tolerate much larger load increment and time step under static and dynamic analyses, respectively, than the two-node thin beam finite element (with translational and rotational dofs).

\section{LINEAR FORMULAION}

In this section, the plane RF beam element formulated very recently by the authors in the course of developing a RF triangle [11] is briefly reviewed. We shall restrict the use of the term "segment" to the beam length between two consecutive nodes along a beam.

\subsection{The RF Element \& Its Interpolation Domain}

As mentioned above, the interpolation domain is generally larger than the integration domain (also called as element domain). For the present RF formulation, the integration domain is a nodebased domain. For a straight beam as shown in Figure 1(a), the integration domain covers the range from the middle point of segment 1-2 to the middle point of segment 2-3, i.e., $\left(X_{1}+X_{2}\right) / 2 \leq X \leq\left(X_{2}\right.$ $\left.+X_{3}\right) / 2$. The corresponding interpolation domain covers the range from Node 1 to 3 . Three nodes are involved and can be used to construct the interpolation.

Similarly, for a curved beam as shown in Figure 2, the integration domain is from the middle point of segment 1-2 to the middle point of segment 2-3 while the interpolation domain covers the range 
from Node 1 to 3 . It is noted that the middle points of curved segments 1-2 and 2-3 are difficult to determine. The curved segments are firstly projected onto the straight line 1-3 and then the middle points of the projected segments are used to help the determination of the integration domain as shown in the figure.

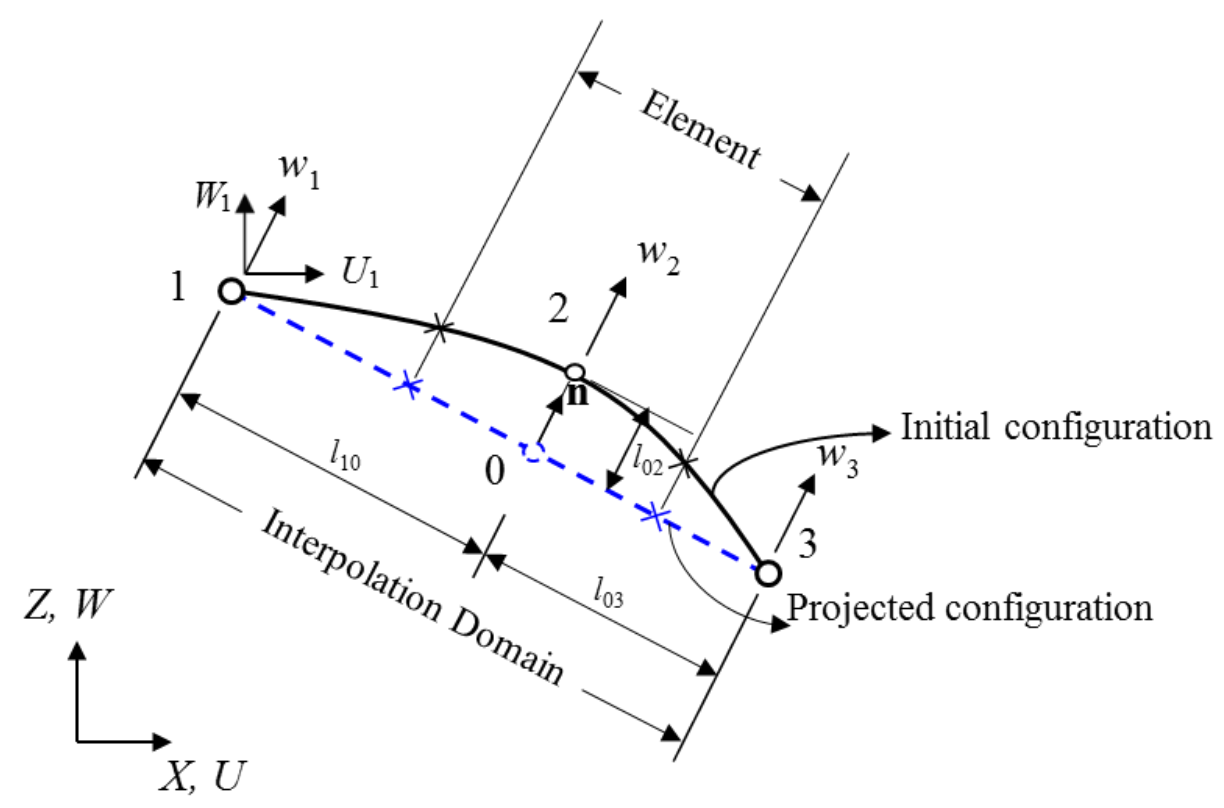

Figure 2. Three consecutive nodes along a curved plane beam.

\subsection{Formulation of Straight RF Beam}

Figure 1(a) shows an initially straight beam. The following quadratic polynomial can be interpolated for the transverse displacement $W$ in the interpolation domain [1], i.e.

$$
W=\frac{\left(X-X_{2}\right)\left(X-X_{3}\right)}{\left(X_{1}-X_{2}\right)\left(X_{1}-X_{3}\right)} W_{1}+\frac{\left(X-X_{1}\right)\left(X-X_{3}\right)}{\left(X_{2}-X_{1}\right)\left(X_{2}-X_{3}\right)} W_{2}+\frac{\left(X-X_{1}\right)\left(X-X_{2}\right)}{\left(X_{3}-X_{1}\right)\left(X_{3}-X_{2}\right)} W_{3}
$$

where $W_{i}$ and $X_{i}$ denote the nodal transverse displacement and the axial coordinate, respectively. Noticeably, the above $W$ is quadratic which is lower than the cubic $W$ employed in the two-node finite element beam model. The curvature can be obtained as:

$$
\kappa=-W,_{X X}=-\frac{2}{l_{13}}\left[\begin{array}{lll}
\frac{1}{l_{12}} & -\left(\frac{1}{l_{12}}+\frac{1}{l_{23}}\right) & \frac{1}{l_{23}}
\end{array}\right]\left\{\begin{array}{l}
W_{1} \\
W_{2} \\
W_{3}
\end{array}\right\}
$$

where $l_{i j}=X_{j}-X_{i}$. The above curvature is used for the node-based element as defined before and the elastic energy in the RF element is 


$$
E^{b}=\frac{E I}{2} l \kappa^{2}=\frac{E I}{2 l}\left\{\begin{array}{l}
W_{1} \\
W_{2} \\
W_{3}
\end{array}\right\}^{T}\left[\begin{array}{ccc}
\frac{1}{l_{12}^{2}} & \frac{-1}{l_{12}}\left(\frac{1}{l_{12}}+\frac{1}{l_{23}}\right) & \frac{1}{l_{12} l_{23}} \\
\frac{-1}{l_{12}}\left(\frac{1}{l_{12}}+\frac{1}{l_{23}}\right) & \left(\frac{1}{l_{12}}+\frac{1}{l_{23}}\right)^{2} & \frac{-1}{l_{23}}\left(\frac{1}{l_{12}}+\frac{1}{l_{23}}\right) \\
\frac{1}{l_{12} l_{23}} & \frac{-1}{l_{23}}\left(\frac{1}{l_{12}}+\frac{1}{l_{23}}\right) & \frac{1}{l_{23}^{2}}
\end{array}\right]\left\{\begin{array}{l}
W_{1} \\
W_{2} \\
W_{3}
\end{array}\right\}
$$

in which $E I$ is the flexural rigidity of the beam and $l=l_{13} / 2$ is the element length. The elastic energy of the beam is obtained by summing the elemental elastic energy.

\subsection{Formulation of Curved RF Beam}

Reference [1] does not extend the above straight beam element to curved beam element which, nevertheless, is considered in Reference [11] by a simple projection scheme. Nodes 1 to 3 in Figure 2 are consecutive nodes along an initially curved beam on the X-Z-plane. Point 0 is the projection of node 2 onto the straight line 1-3 and $\mathbf{n}$ is the unit vector perpendicular to 1-3. Provided that 1-2-3 remains shallow, the projected coordinates can be used to interpolate the initial and deformed configurations. The curvature change can be obtained in a way similar to that of Eq.(2) as:

$$
\kappa=-\frac{2}{l_{13}}\left[\frac{1}{l_{10}}-\left(\frac{1}{l_{10}}+\frac{1}{l_{03}}\right) \frac{1}{l_{03}}\right]\left(\left\{\begin{array}{c}
\mathbf{n}^{T} \mathbf{U}_{1} \\
\mathbf{n}^{T} \mathbf{U}_{2}+l_{02} \\
\mathbf{n}^{T} \mathbf{U}_{3}
\end{array}\right\}-\left\{\begin{array}{c}
0 \\
l_{02} \\
0
\end{array}\right\}\right)=-\frac{2}{l_{13}}\left[\begin{array}{ccc}
\frac{1}{l_{10}} & -\left(\frac{1}{l_{10}}+\frac{1}{l_{03}}\right) & \frac{1}{l_{03}}
\end{array}\right] \mathbf{N}\left\{\begin{array}{c}
\mathbf{U}_{1} \\
\mathbf{U}_{2} \\
\mathbf{U}_{3}
\end{array}\right\}
$$

in which

$$
\mathbf{N}=\left[\begin{array}{lll}
\mathbf{n}^{T} & & \\
& \mathbf{n}^{T} & \\
& & \mathbf{n}^{T}
\end{array}\right] \text { and } \mathbf{U}_{i}=\left\{\begin{array}{l}
U_{i} \\
W_{i}
\end{array}\right\} \text { is nodal displacement vector. }
$$

In the equation, $\mathbf{n}^{T} \mathbf{U}_{i}$ is equivalent to transverse displacement $W_{i}$ in Eq.(2). The bending energy of the element associated with Node 2 is

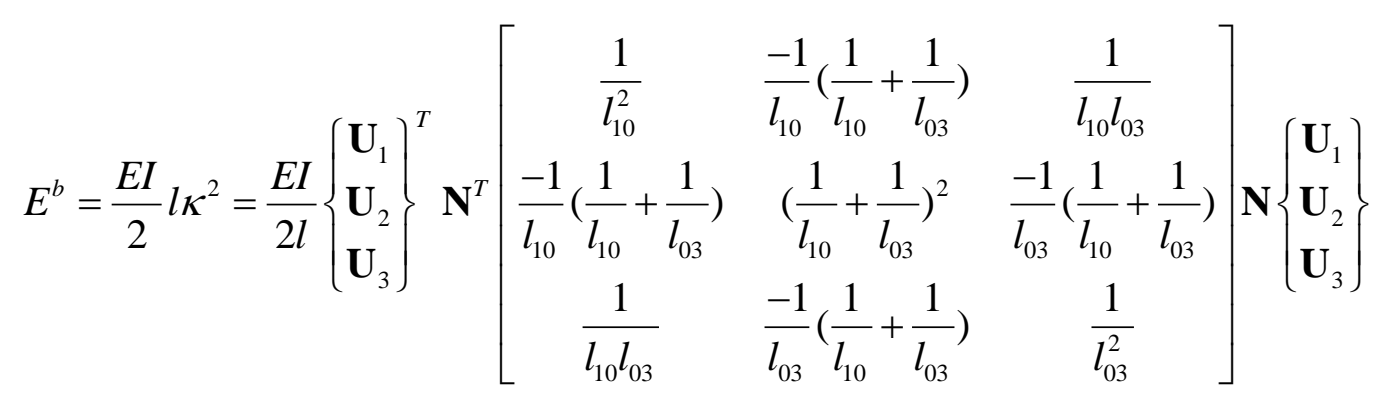

where the element length $l$ is taken to be $l_{13} / 2$. To take the stretching energy into account, the conventional two-node truss finite element is used. 


\subsection{Imposition of Boundary Conditions}

The treatment on the boundary conditions with RF element is not as obvious as that with traditional beam element, since the adjacent nodes of the boundary elements may not exist. In the current approach, slave fictious nodes are employed to impose the boundary conditions similarly as done by Phaal \& Calladine [1]. Two cases, i.e., symmetric and free conditions, are considered. The clamped conditions can be modeled by applying the symmetric conditions and by fixing the nodal displacement while the simply supported conditions can be modeled by applying the free conditions and by fixing the nodal displacement.

For the symmetric boundary condition as shown in Figure 3, a slave node 1', which is the symmetric point of node 1 corresponding to the symmetric boundary, is firstly generated. The displacements of the slave node 1' can be determined according to the symmetric condition, i.e., $U_{1}=-U_{1}$, and $W_{1}=W_{1}$. For the free edge condition, it means that the moment is null. Thus, the bending energy is also null.

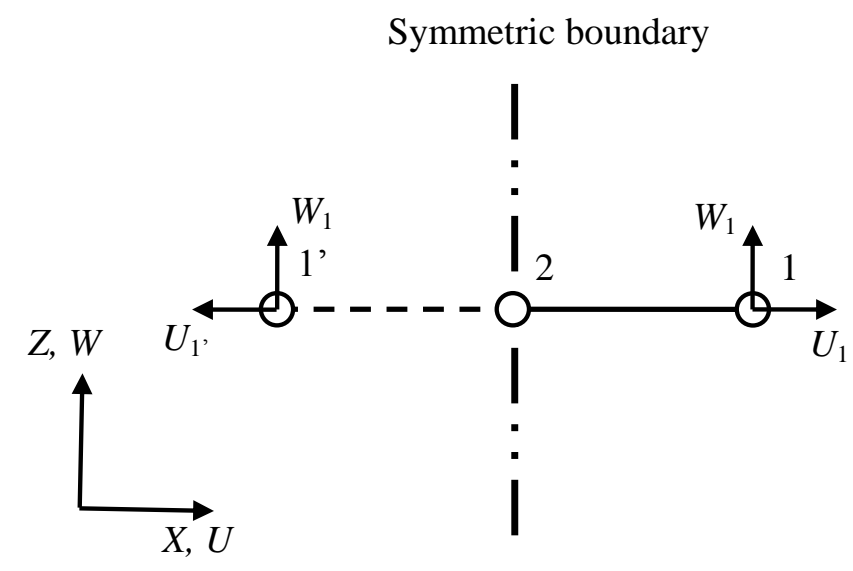

Figure 3. Imposition of symmetric boundary condition

\section{LINEAR EXAMPLES}

The focus of the previous paper [11] is the shell element formulation and only one example on a linear beam is presented. Here, two straight beam examples previously considered by Flores \& Oñate [8] are examined to illustrate the efficacy of the present formulation.

\subsection{Simple supported straight beam}

This simply supported straight beam is loaded by uniform transverse load as shown in Figure 4(a). The distributed load over an element is lumped and taken to be the nodal force applied to the central node of the element. Owing to symmetry, half of the beam is modeled with 1,2, and 4 equal segments. The normalized nodal deflections with respect to the exact midspan deflection are shown 
in Figure 4(b). It should be remarked that the nodal predictions of Flores \& Oñate [8] are computed from the reported deflections at the midpoints of the segments which are taken to be straight in their formulation. Compared with Flores \& Oñate's beam element, the present one yields more accurate predictions in this example.

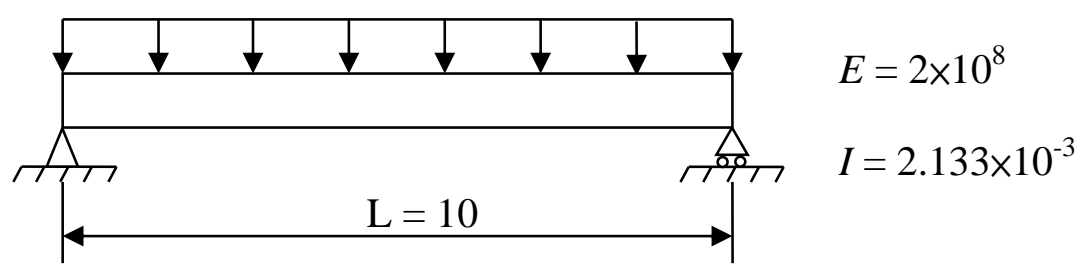

(a)

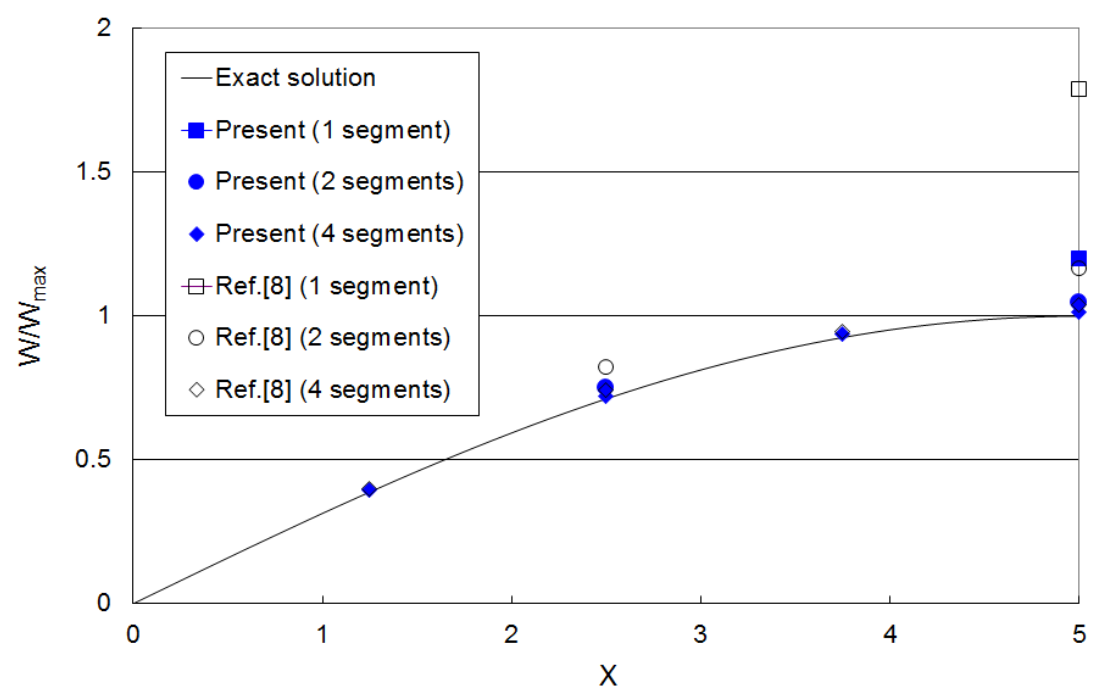

(b)

Figure 4 (a) Simple supported plane beam under uniform load. (b) The normalized deflections along the beam.

\subsection{Cantilever beam}

This simple cantilever beam is subjected to a point moment load as shown in Figure 5(a). Since there is no rotational degree of freedom with RF element, the point moment load cannot be directly applied. However, an equivalent force couple can be applied to the adjacent nodes to replace the point moment as shown in Figure 5(b), where $h$ is the segment length and two nodal forces $P=M / h$ are used to construct the force couple. For the present example, the bending moment is constant throughout the whole beam which should be exactly represented by the RF elements. Thus, the error if exists should be resulted from the load treatment. The beam is modeled with uniform segments. Fortunately, the results show that no matter how many segments are used, the numerical model can achieve the exact solution. Therefore, using the equivalent force couple to replace the point moment is reasonable. 


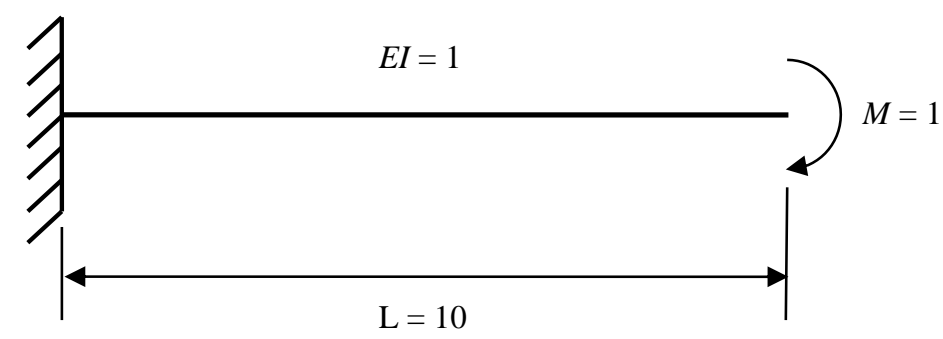

(a)

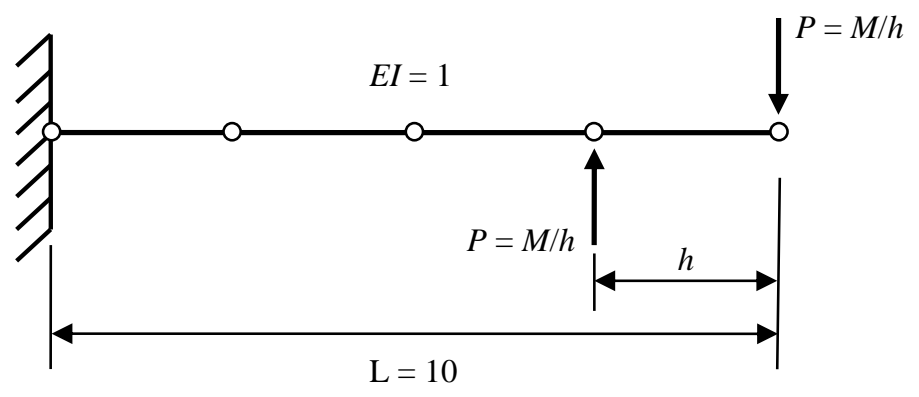

(b)

Figure 5 (a) Cantilever beam subjected to point moment load. (b) Cantilever beam subjected to equivalent force couple.

\subsection{L-frame}

The lower and upper ends of this L-frame are built-in and simply supported as shown in Figure 6(a). At the junction B of the horizontal and vertical branches, a horizontal force is applied. This example has also been considered by Flores \& Oñate [8]. They did not mention the tensile rigidity EA of the two branches. For simplicity, their cross-sections are treated as circular here. In this light, their diameter-to-length ratios are around 17. Two schemes are employed to consider the junction. The first scheme models the whole frame as a single smooth beam. In other words, the junction is approximated as an arc and a curved RF beam element is used across the junction, which is also the practice of Flores \& Oñate [8]. The second scheme models the horizontal and vertical branches as individual straight beams whilst the equal-rotation and moment balance conditions at $\mathrm{B}$ are enforced. Each branch is modeled by 3, 6 and 12 equal segments. The horizontal nodal deflections along the vertical branch are normalized by the thin-beam solution of the horizontal deflection at the junction and shown in Figure 6(b). In the figure, "Present 1" and "Present 2" refer to the predictions using the first and second modeling schemes, respectively. Comparing "Present 1" with the prediction of Flores \& Oñate [8], the former possesses a higher coarse-mesh accuracy. Using 12 segments per branch, the former and the latter are slightly smaller and larger than the thin-beam solution. "Present 2" is roughly the average of "Present 1" and the prediction of Flores \& Oñate [8]. 
Moreover, "Present 2" overlaps with the thin-beam solution when 12 segments per branch are employed. The example is also considered by Abaqus. Using a large number of B21 elements, the horizontal deflection at $\mathrm{B}$ is $0.6 \%$ larger than that from the thin-beam solution. The Abaqus prediction is graphically coincident with Present 2 using 12 segments.

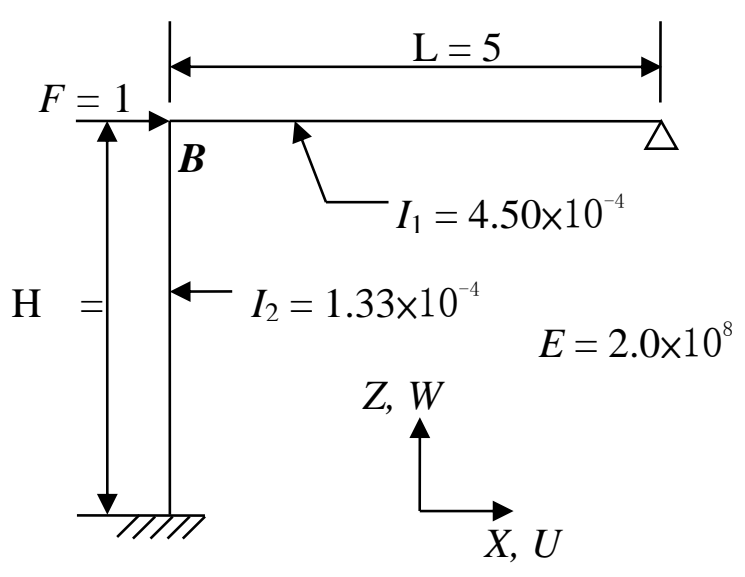

(a)

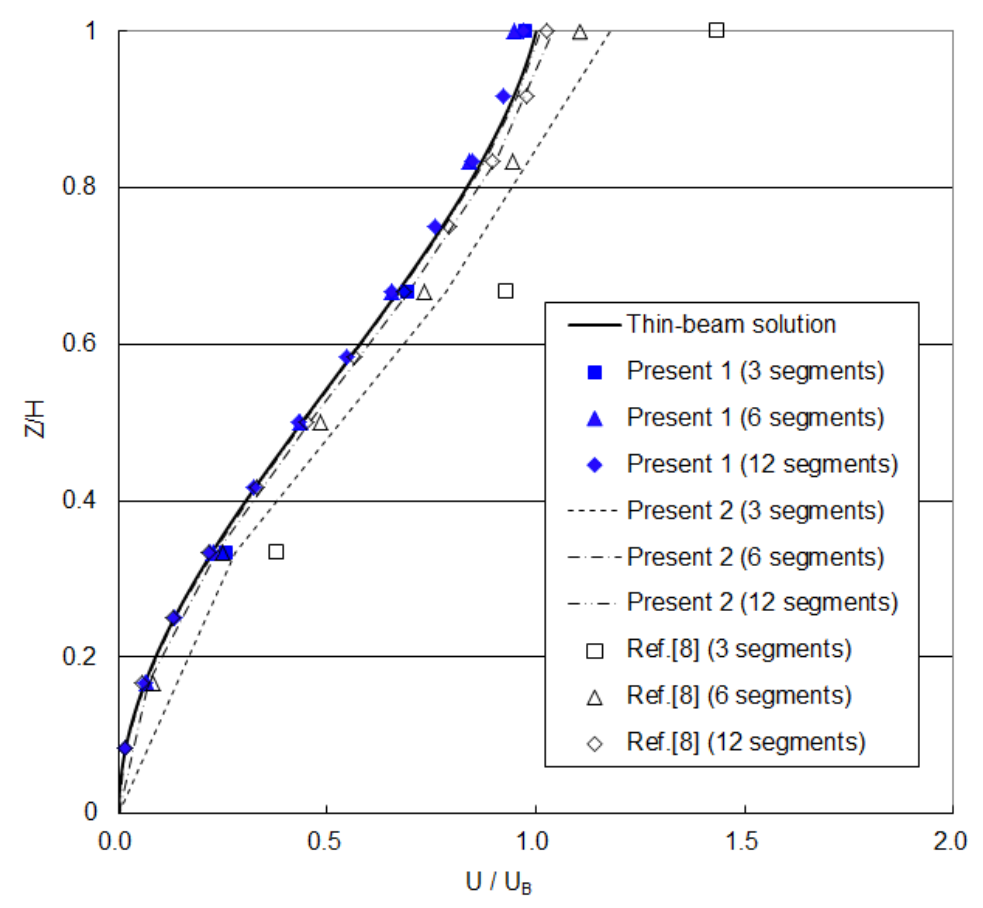

(b)

Figure 6 (a) A L- frame acted upon by a horizontal force. (b) Normalized horizontal displacements along the vertical member.

\section{NONLINEAR FORMULAION}

In this section, the linear formulation is extended to the geometrically nonlinear analysis using a corotational approach. It will be seen that the tangent bending stiffness matrix for the initially straight beam is constant and does not need updating. For the curved beam, the tangent bending stiffness 
matrix can also be approximated as constant.

\subsection{Formulation of Straight Beam}

The straight beam formulation is the same as that by Sze \& Liu [24]. It is briefly described for completeness. Consider Nodes 1, 2 and 3 along the initial straight beam in Figure 7. $\mathbf{U}_{1}, \mathbf{U}_{2}$ and $\mathbf{U}_{3}$ are the displacement vectors of Nodes 1,2 and 3, respectively, and the deformed configuration is 1'-2'-3'. A co-rotated $\mathrm{x}-\mathrm{z}$-frame is defined such that its origin is 1' and the $\mathrm{x}$-axis passes thru 3'. There exists a rigid body displacement $\mathbf{U}^{C}$ which brings $1-2-3$ to its co-rotated configuration $1^{\prime}-2^{C}-$ $3^{\mathrm{C}}$. It is trivial that $\mathbf{U}^{C}$ is a linear function of $x$, i.e.

$$
\mathbf{U},{ }_{x x}^{C}=\mathbf{0} .
$$

Provided that the axial strain and curvature is small, the displacement from the co-rotated configuration to the deformed configuration is small and closely parallel to the $z$-axis which defined the unit vector $\mathbf{n}$. Thus, the local transverse displacement $w$ can be obtained as:

$$
w \simeq \mathbf{n}^{T}\left(\mathbf{U}-\mathbf{U}^{C}\right)
$$

and

$$
\mathbf{U}-\mathbf{U}^{C} \simeq \mathbf{n} w \simeq \mathbf{n n}^{T}\left(\mathbf{U}-\mathbf{U}^{C}\right) .
$$

Similar to Eq.(2) and Eq.(3), the bending energy can be quantified as:

$$
E^{b}=\frac{E I}{2} l \kappa^{2}=\frac{E I}{2} l\left(w,{ }_{x x}\right)^{2}=\frac{E I}{2} l\left(\mathbf{U}-\mathbf{U}^{C}\right){ }_{x x}^{T} \mathbf{n n}^{T}\left(\mathbf{U}-\mathbf{U}^{C}\right){ }_{x x}
$$

in which, $l$ is taken to be $l_{13} / 2$. By virtue of Eq.(6) and Eq.(8), the above equation can be simplified as:

$$
E^{b}=\frac{E I}{2} l \mathbf{U},_{x x}^{T} \mathbf{n n}^{T} \mathbf{U},_{x x} \simeq \frac{E I}{2} l \mathbf{U}_{x x}^{T} \mathbf{U},_{x x} .
$$

As $\mathbf{U}$ can be interpolated as a quadratic function of $x$,

$$
\mathbf{U}_{x x}=\frac{2}{l_{12}+l_{23}}\left[\frac{1}{l_{12}} \mathbf{I}_{2}-\left(\frac{1}{l_{12}}+\frac{1}{l_{23}}\right) \mathbf{I}_{2} \frac{1}{l_{23}} \mathbf{I}_{2}\right]\left\{\begin{array}{l}
\mathbf{U}_{1} \\
\mathbf{U}_{2} \\
\mathbf{U}_{3}
\end{array}\right\}=\mathbf{B} \mathbf{U}_{1.3}
$$

where $\mathbf{B}$ is self-defined. The bending energy and its derivatives required for the nonlinear iterations can then be derived as:

$$
E^{b}=\frac{l_{13}}{2} \frac{E I}{2} \mathbf{U}_{1 \ldots 3}^{T} \mathbf{B}^{T} \mathbf{B} \mathbf{U}_{1 \ldots 3}, \frac{\partial E^{b}}{\partial \mathbf{U}_{1 \ldots 3}}=\frac{l_{13}}{2} E I \mathbf{B}^{T} \mathbf{B} \mathbf{U}_{1 \ldots 3}, \frac{\partial}{\partial \mathbf{U}_{1 \ldots 3}}\left(\frac{\partial E^{b}}{\partial \mathbf{U}_{1 \ldots 3}}\right)^{T}=\frac{l_{13}}{2} E I \mathbf{B}^{T} \mathbf{B}
$$

The first and second derivatives with respective to the vector of nodal displacements $\mathbf{U}_{1 . .3}$ are the 
internal force vector and the tangent bending stiffness matrix, respectively. It can be seen that the latter is a constant matrix which does not require updating in the iterative solution process. To take the axial effect into account, the simple two-node truss element formulated under the total Lagrangian framework is employed.

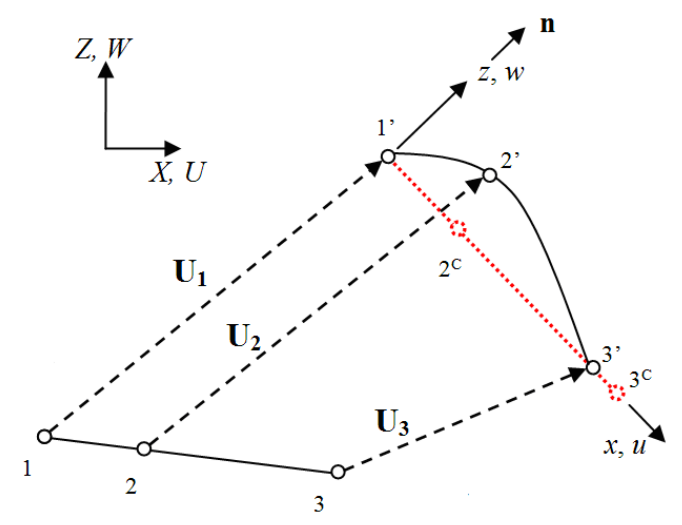

Figure 7. An initially straight beam segment in nonlinear analysis. $1-2-3,1{ }^{\prime} 2^{\mathrm{C}} 3^{\mathrm{C}}$ and $1^{\prime}-2$ '- -3 ' are the initial, corotational and deformed configurations, respectively.

\subsection{Formulation of Curved Beam}

The extension from linear to nonlinear formulation of the curved beam is analogous to that of the straight beam. Considering Figure 8, 1-2-3, 1'-2'-3' and $1^{\prime}-2^{\mathrm{C}}-3^{\mathrm{C}}$ are the initial, deformed and corotated configurations, respectively. Again, the origin of the co-rotated $x-y$-frame is 1' with the $x$ axis passing thru 3' and $\mathbf{n}$ being the unit vector along the z-axis. Similar to Eq.(4), the curvature change is

$$
\kappa=-\frac{2}{l_{10}+l_{03}}\left[\begin{array}{lll}
\frac{1}{l_{10}} & -\left(\frac{1}{l_{10}}+\frac{1}{l_{03}}\right) & \frac{1}{l_{03}}
\end{array}\right]\left\{\begin{array}{l}
\mathbf{n}^{T}\left(\mathbf{U}_{1}-\mathbf{U}_{1}^{C}\right) \\
\mathbf{n}^{T}\left(\mathbf{U}_{2}-\mathbf{U}_{2}^{C}\right) \\
\mathbf{n}^{T}\left(\mathbf{U}_{3}-\mathbf{U}_{3}^{C}\right)
\end{array}\right\}=-\mathbf{L}\left\{\begin{array}{l}
\mathbf{n}^{T}\left(\mathbf{U}_{1}-\mathbf{U}_{1}^{C}\right) \\
\mathbf{n}^{T}\left(\mathbf{U}_{2}-\mathbf{U}_{2}^{C}\right) \\
\mathbf{n}^{T}\left(\mathbf{U}_{3}-\mathbf{U}_{3}^{C}\right)
\end{array}\right\}
$$

in which $\mathbf{U}^{C}$ is the rigid body co-rotational displacement and $\mathbf{L}$ is self-defined. Let 0 and $0^{C}$ be the projections of 2 and $2^{C}$ onto 1-3 and 1'-3 ${ }^{C}$, respectively. Then, $\left|\mathbf{X}_{02}\right|=\left|\mathbf{X}_{0^{C} 2^{C}}\right|=\mathbf{n}^{T} \mathbf{X}_{0^{C} 2^{C}}$ where $\mathbf{X}_{i j}=$ $\mathbf{X}_{j}-\mathbf{X}_{i}$ and

$$
\begin{aligned}
& \mathbf{L}\left\{\begin{array}{l}
\mathbf{n}^{T} \mathbf{U}_{1}^{C} \\
\mathbf{n}^{T} \mathbf{U}_{2}^{C} \\
\mathbf{n}^{T} \mathbf{U}_{3}^{C}
\end{array}\right\}=\mathbf{L}\left\{\begin{array}{c}
\mathbf{n}^{T} \mathbf{U}_{1}^{C} \\
\mathbf{n}^{T}\left(\mathbf{U}_{0}^{C}+\mathbf{X}_{0^{C} 2^{C}}-\mathbf{X}_{02}\right) \\
\mathbf{n}^{T} \mathbf{U}_{3}^{C}
\end{array}\right\}=\mathbf{L}\left\{\begin{array}{c}
\mathbf{n}^{T} \mathbf{U}_{1}^{C} \\
\mathbf{n}^{T} \mathbf{U}_{0}^{C} \\
\mathbf{n}^{T} \mathbf{U}_{3}^{C}
\end{array}\right\}+\mathbf{L}\left\{\begin{array}{c}
0 \\
\mathbf{n}^{T} \mathbf{X}_{0^{C} 2^{C}} \\
0
\end{array}\right\}-\mathbf{L}\left\{\begin{array}{c}
0 \\
\mathbf{n}^{T} \mathbf{X}_{02} \\
0
\end{array}\right\} \\
& =\mathbf{L}\left\{\begin{array}{c}
0 \\
\left|\mathbf{X}_{02}\right| \\
0
\end{array}\right\}-\mathbf{L}\left\{\begin{array}{c}
0 \\
\mathbf{n}^{T} \mathbf{X}_{02} \\
0
\end{array}\right\}=-\kappa_{0}-\mathbf{L}\left\{\begin{array}{c}
0 \\
\mathbf{n}^{T} \mathbf{X}_{02} \\
0
\end{array}\right\}
\end{aligned}
$$

where the underlined term vanishes by virtue of Eq.(6); $\kappa_{0}$ is self-defined and is the initial curvature. 
Substitution of Eq.(14) into Eq.(13) yields

$$
\boldsymbol{\kappa}=-\mathbf{L}\left\{\begin{array}{c}
\mathbf{n}^{T} \mathbf{U}_{1} \\
\mathbf{n}^{T}\left(\mathbf{U}_{2}+\mathbf{X}_{02}\right) \\
\mathbf{n}^{T} \mathbf{U}_{3}
\end{array}\right\}-\kappa_{o}=\mathbf{n}^{T} \tilde{\mathbf{B}}\left\{\begin{array}{c}
\mathbf{U}_{1} \\
\mathbf{U}_{2}+\mathbf{X}_{02} \\
\mathbf{U}_{3}
\end{array}\right\}-\kappa_{o}=\mathbf{n}^{T} \tilde{\mathbf{B}}\left\{\begin{array}{c}
\mathbf{U}_{1} \\
\mathbf{U}_{2}+\mathbf{X}_{02} \\
\mathbf{U}_{3}
\end{array}\right\}-\kappa_{o}
$$

in which $\tilde{\mathbf{B}}$ can be obtained from $\mathbf{B}$ in Eq.(11) by replacing $l_{12}$ with $l_{10}$ and replacing $l_{23}$ with $l_{03}$. Hence, the bending energy associated with Node 2 is

$$
E^{b}=\frac{l_{13}}{2} \frac{E I}{2} \kappa^{2}=\frac{l_{13}}{2} \frac{E I}{2}\left(\mathbf{n}^{T} \tilde{\mathbf{B}}\left\{\begin{array}{c}
\mathbf{U}_{1} \\
\mathbf{U}_{2}+\mathbf{X}_{02} \\
\mathbf{U}_{3}
\end{array}\right\}-\kappa_{o}\right)^{2}
$$

and the corresponding internal force is

$$
\frac{\partial E^{b}}{\partial \mathbf{U}_{1.3}}=\frac{l_{13}}{2} m\left(\tilde{\mathbf{B}}^{T} \mathbf{n}+\frac{\partial \mathbf{n}^{T}}{\partial \mathbf{U}_{1 . .3}} \tilde{\mathbf{B}}\left\{\begin{array}{c}
\mathbf{U}_{1} \\
\mathbf{U}_{2}+\mathbf{X}_{02} \\
\mathbf{U}_{3}
\end{array}\right\}\right)
$$

where

$$
m=E I\left(\mathbf{n}^{T} \tilde{\mathbf{B}}\left\{\begin{array}{c}
\mathbf{U}_{1} \\
\mathbf{U}_{2}+\mathbf{X}_{02} \\
\mathbf{U}_{3}
\end{array}\right\}-\kappa_{o}\right) \text { is the bending moment. }
$$

The explicit expression of the derivative of $\mathbf{n}$ is rather lengthy. Nevertheless, there is only one directional vector in the present formulation while more directional vectors are involved in other RF element formulation. The present one is obviously the simplest.
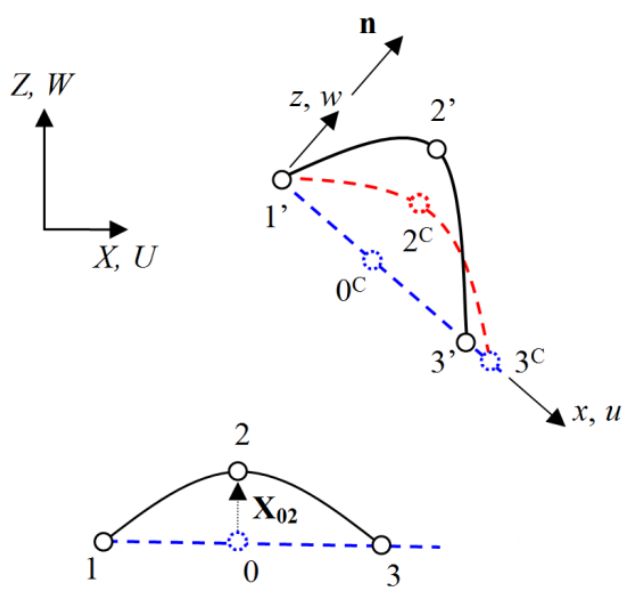

Figure 8. Initial configuration, deformed configuration, and corotational configuration of a curved beam.

The tangent bending stiffness matrix can be obtained as the second order derivative of the bending energy. The explicit expression of the matrix would be extremely lengthy due to the complexity of the second order derivative of $\mathbf{n}$. In the previous paper [11], a simplification by 
assuming 1-2-3 to be initially straight or $\kappa_{o}$ equal to zero is adopted such that the second derivative of the energy is approximated by the following constant matrix:

$$
\frac{\partial}{\partial \mathbf{U}_{1 \ldots 3}}\left(\frac{\partial E^{b}}{\partial \mathbf{U}_{1 \ldots 3}}\right)^{T} \simeq \frac{l_{13}}{2} E I \tilde{\mathbf{B}}^{T} \tilde{\mathbf{B}}
$$

which, same as that in Eq.(12) for straight beam, requires no updating in the iterative solution process. It should be remarked that the simplification $\kappa_{o}=0$ should not be adopted in deriving the first derivative of the energy or the internal force vector which determines the deformed configuration in response to the external force. In examples considered in Reference [24], the collectively adverse effect of the simplification on the convergence rate is minimal.

\section{NONLINEAR EXAMPLES}

Two straight and one curved beam examples have been presented in References [24] and [11], respectively. In this section, two additional examples previously considered by other RF elements are examined. The $0.5 \%$ force tolerance is employed as the convergence criterion, i.e.

$$
\frac{\mid \text { global external force vector }- \text { global internal force vector } \mid}{\mid \text { global external force vector } \mid}<0.5 \%
$$

in which $\|$ returns the magnitude of the embraced vector.

\subsection{Z-shaped Cantilever}

Figure 9(a) shows a Z-shaped cantilever subjected to a vertical point force at the free end. It contains two horizontal and one inclined branches. The cross section is rectangular. Following the practice of Flores \& Oñate [8] and Battini [10], the cantilever is modeled as a continuous beam, i.e. the sharp bends at 60 and 120 units from the clamped end are treated as arcs with their initial curvatures determined by the nodal spacing. The curves of load versus vertical deflection at the tip yielded by using 9 and 18 segments are plotted in Figure 9(b). The reference solution is a highly converged one computed by using a large number of Abaqus's two-node plane beam element B21. It can be seen that the present results are satisfactory. Furthermore, the free end vertical deflections at $F=1263$ (in the non-linear region) for different numbers of segment are compared with those obtained by Flores \& Oñate [8] and Battini [10] in Table 1 after normalized with 133.09 which is the reference solution used by Battini. The normalized results are listed in Table 1. All RF elements yield close predictions. The present element and that of Flores \& Oñate [8] yield nearly identical predictions. They are less accurate than that of Battini [10]. Nevertheless, the present formulation is far simpler than those of the other two RF elements. 


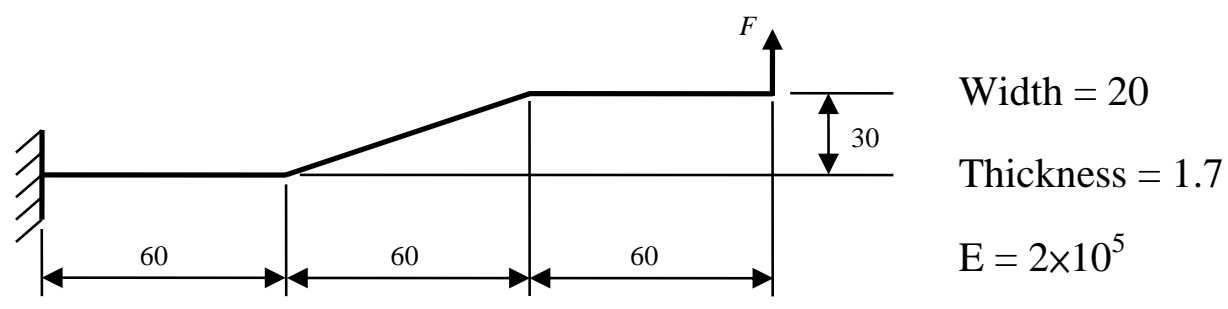

(a)

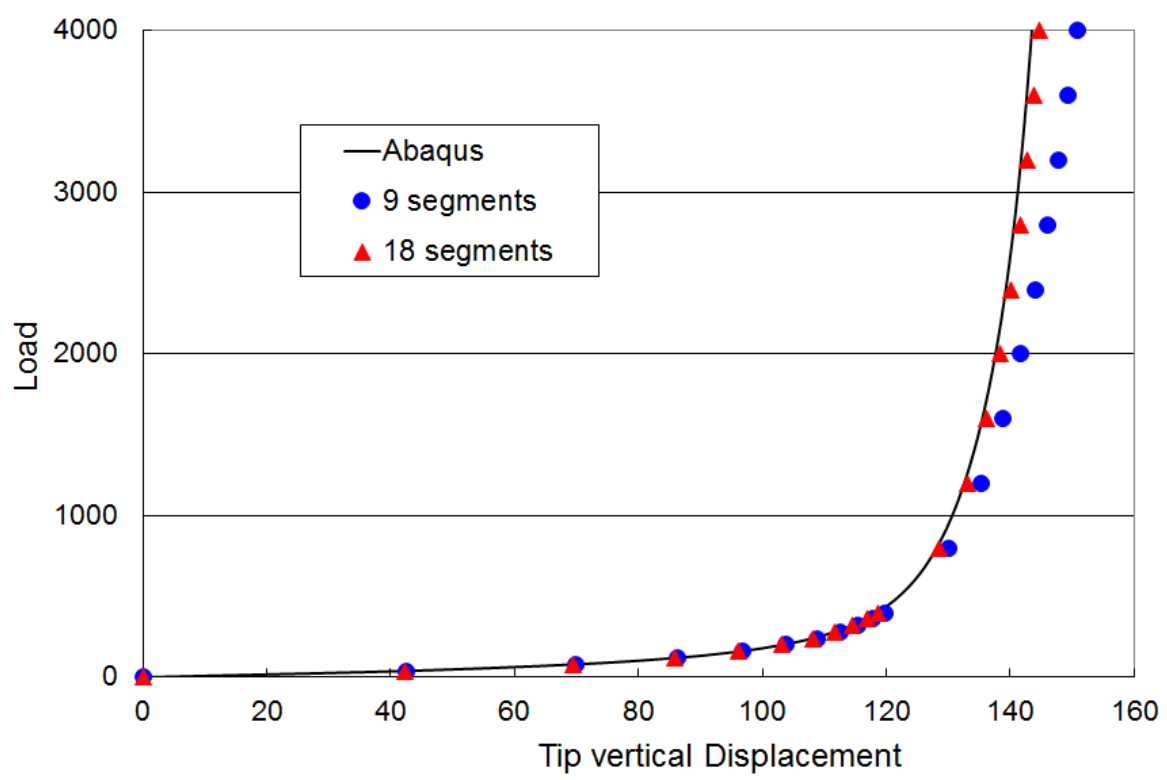

Figure 9. (a) The Z-shaped cantilever loaded by a vertical point force. (b) Load versus the tip vertical deflection.

Table 1. Normalized vertical free-end displacement at $\mathrm{P}=1263$.

\begin{tabular}{|c|c|c|c|}
\hline Number of segments & 18 & 9 & 6 \\
\hline Present & 1.005 & 1.021 & 1.056 \\
\hline Flores \& Oñate [8] & 1.01 & 1.03 & 1.06 \\
\hline Battini [10] & 0.999 & 0.997 & 0.997 \\
\hline
\end{tabular}

\subsection{Built-in Arch}

Figure 10(a) shows a circular arch with both ends built-in. It is acted upon by a downward force $\mathrm{P}$ at its apex B. The force is plotted against the downward deflection $d$ of $\mathrm{B}$ in Figure 10(b). A limit point can be seen in the curve and displacement loading is employed to avoid the instability in the numerical solution. The loading stops at $d=120$ which exceeds the radius of the arch. The prediction of Battini's RF beam element [10] is included and the reference solution is the highly converged one obtained by using 200 B21 elements which are capable of taking the initial curvature into account. Using 40 segments, the element of Battini yields more accurate prediction than the present one. By doubling the number of segments, the error of the present element is roughly halved. The present element continues to converge to reference solution with more segments. The 
results, however, are not plotted for graphical clarity.

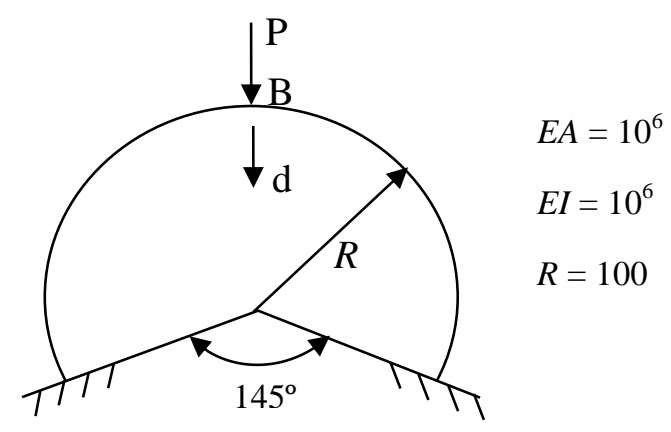

(a)

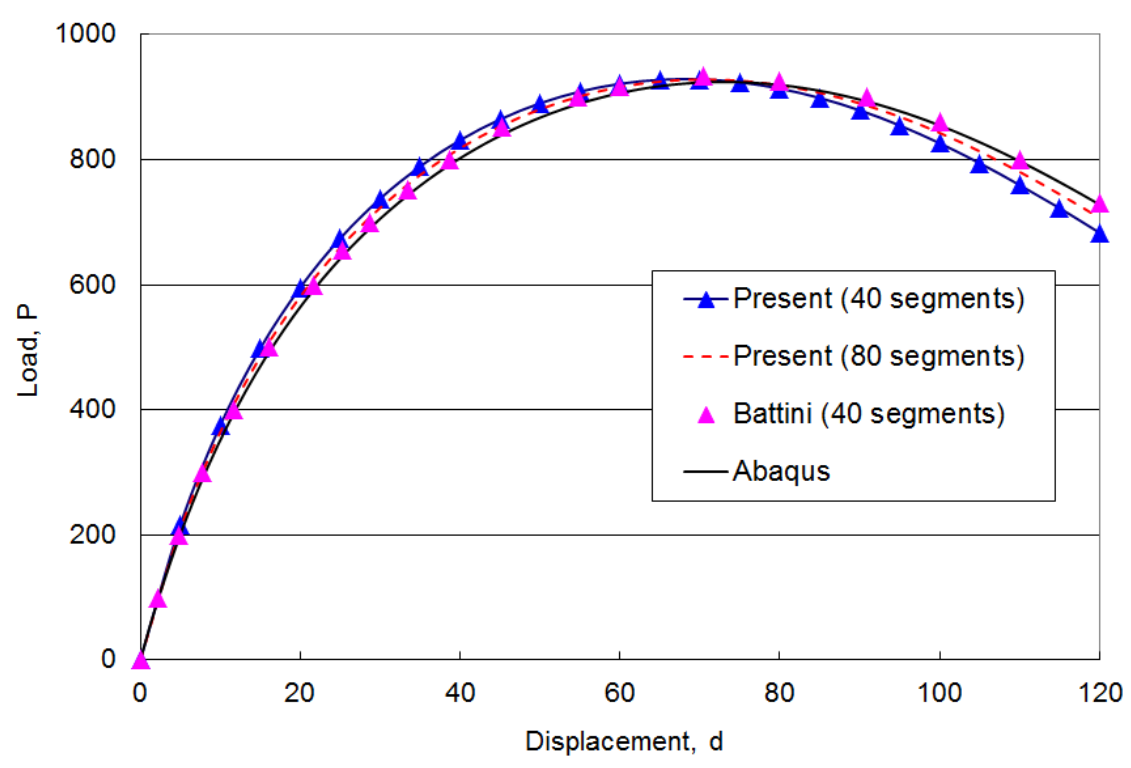

(b)

Figure 10 (a) A circular arch loaded by a point force. (b) The load is plotted against the deflection under the load.

\section{PSUEDO THREE-DIMENSIONAL BEAM FOR CABLE ANALYSIS}

In the previous sections, a plane beam is considered. For 3D beam analyses, bending effect about two mutually perpendicular transverse axes and torsional effect about the longitudinal axis should be considered. To the best of our knowledge, there has not been a 3D RF beam element. It is indeed impossible for the RF beam to consider the torsional effect due to the absence of rotational dofs. For cable analyses, a comprehensive beam formulation may not be necessary as the bending and torsional rigidities of the cable are relatively small compared with the axial stiffness; the cross section is usually isotropic; and the cable should be initially straight. Here, the torsional effect is neglected but the bending effect is retained. With the initial straight configuration, the derivation for the geometric nonlinear plane beam in Section 4.1 is largely applicable. With reference to Figure 7, 
one only needs to add another dimension ( $\mathrm{Y}$, and the displacement $\mathrm{V}$ along it) and aligns the $z$-axis (and the unit 3D vector $\mathbf{n}$ along it) such that the x-z-plane is coplanar with 1'-2'-3'. Eq.(11) should accordingly be generalized to

$$
\mathbf{U},_{x x}=\left\{\begin{array}{l}
U,_{x x} \\
V,{ }_{x x} \\
W,{ }_{x x}
\end{array}\right\}=\frac{1}{l_{12}+l_{23}}\left[\frac{1}{l_{12}} \mathbf{I}_{3}-\left(\frac{1}{l_{12}}+\frac{1}{l_{23}}\right) \mathbf{I}_{3} \frac{1}{l_{23}} \mathbf{I}_{3}\right]\left\{\begin{array}{l}
\mathbf{U}_{1} \\
\mathbf{U}_{2} \\
\mathbf{U}_{3}
\end{array}\right\}
$$

With Eq.(20) substituted into Eq.(10) and Eq.(12), the rank of the constant matrix arising from the last expression in Eq.(12) is 3. Again, the two-node 3D truss finite element formulated under the total Lagrangian framework is employed to account for the axial effect.

To give some physical insight on the bending energy in Eq.(10) under the 3D setting and the limitation of the present 3D RF cable element, the bending energy for three-dimensional displacement can be expanded as:

$$
E^{b}=\frac{E I}{2} l\left(U,_{x x}^{2}+V,_{x x}^{2}+W,_{x x}^{2}\right)
$$

In the expression, $V, x x$ and $W, x x$ are the curvatures in respectively the corotational $x-y$ and the $x-z$ planes whilst $U_{, x x}$ is indeed a negligible error term in the bending energy since the axial strain $U,_{x}$ is small. For isotropic cross-section, the energy and, thus, the tangential stiffness matrix manage to consider the two mutually perpendicular bending moments.

A concern of finite element analysis of large displacement/rotation problems is that the element develops spurious internal force or energy under finite rigid body motions. To this end, a rotationfree 3D curved beam modeled by two node-to-node segments is prescribed with the six independent rigid body modes at finite magnitudes. No internal force or energy develops in the beam.

\section{EXAMPLES ON CABLES}

In this section, the RF cable element will be employed to consider a number of static and dynamic cable problems in References $[15,17]$ in which only the tensile rigidity $E A$ is considered. In the RF cable element, one also needs to specify the bending stiffness which is highly dependent on its wire distribution, friction and loading of the cable. By assuming the closely-packed distribution for seven wires, two bounding values for the bending rigidity can be computed using the formulae in Reference [25] and the approximate average is

$$
E I=0.6 \frac{E A^{2}}{4 \pi}
$$

which will be adopted in all the subsequent examples. Some of them will also be considered by Abaqus's B21 or B31 two-node 2D and 3D thin beam elements, respectively. The torsional stiffness 
of the B31 element will be specified as:

$$
E J=2 E I=1.2 \frac{E A^{2}}{4 \pi} .
$$

In the last two equations, $A$ stands for the nominal cross-sectional area of the cable.

Same as Section 3.1, the distributed load over a RF element is lumped and taken to be the nodal force applied to the central node of the element. In dynamic analyses, the distributed mass is lumped similarly. Hence, the system mass matrix is diagonal which is a property needed for explicit time integration.

\subsection{Cable under Gravity and Point Loads}

Figure 11 shows a cable anchored at two leveled points 1 and 2 which are $304.8 \mathrm{~m}$ apart. The example has been considered in Reference [15]. The unstretched length, cross-section area $A$ and tensile rigidity $E A$ of the cable are $312.73 \mathrm{~m}, 548.4 \mathrm{~mm}^{2}$ and $71.840 \mathrm{MN}$, respectively. The gravity load is $q=46.12 \mathrm{~N} / \mathrm{m}$ and a point force $P=35.586 \mathrm{kN}$ is also applied to point 3 . The unstretched lengths of 1-3 and 3-2 are 125.88 and $186.85 \mathrm{~m}$, respectively. In the computation, the numbers of segment in 1-3 and 3-2 are in the ratio of 2:3 and only the total number segments will be mentioned in the results. Initially, the cable is horizontal and unstretched with its left end attached to point 1. Then, the right end is moved to point 2 whilst the loads $q$ and $P$ are also applied. The cable is modeled by 5, 10 and 20 segments or the same numbers of B31 elements. Besides 45 iterations are allowed per load increment, the load increment and iteration strategies are the same as the default settings of Abaqus for nonlinear static analyses. In essence, the convergence criterion is the simultaneous fulfillment of the $0.5 \%$ force and $1 \%$ displacement tolerance conditions. Moreover, a load increment will be aborted and equally subdivided into four unless the force residue starts to diminish from the fourth iteration onwards and the convergence criterion is met within 45 iterations; or into two unless the projected force residue can meet the force tolerance requirement before the 45-th iteration from the eighth iteration onwards. On the other hand, if the solution converges within five iterations in two consecutive load increments, the next load increment will be increased to 1.5 times of the present load increment.

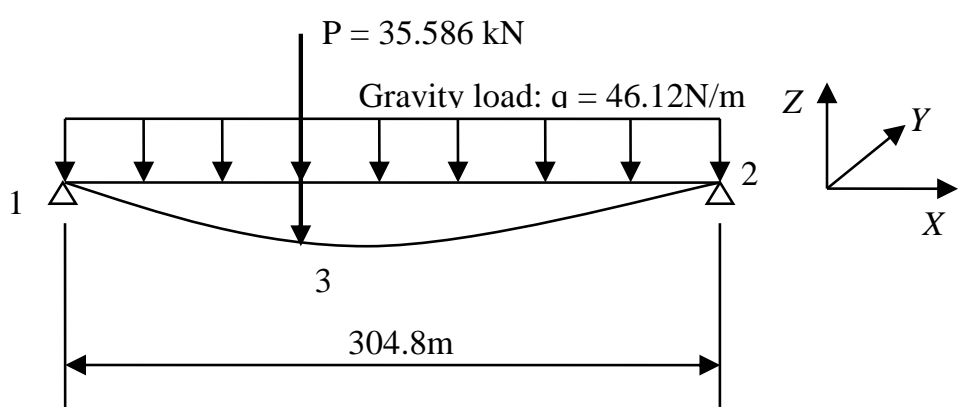


Figure 11. Cable under gravity and a point loads.

In this example, the RF cable method does not require any sub-division in the load increment. On the other hand, the overall load increment in Abaqus is successively reduced to its $10^{-6} \sim 10^{-8}$ times before the first converged solution can be yielded. Table 2 shows the numbers of load increments and iterations employed before the final loads can be applied. Table 3 shows the final converged displacement components of point 3 which is under the point force. The prediction yielded by using 100 Abaqus elements can be taken as the reference solution. Both the present method and Abaqus yield accurate predictions. However, the former appears to have a higher coarse-mesh accuracy and consumes far less load increments/iterations than the latter. Table 3 also gives the predictions of the catenary and truss elements [15] which ignore the bending and torsional rigidities.

Table 2. Comparisons of number of load increments and iterations.

\begin{tabular}{|l|c|c|c|c|c|c|}
\hline \multirow{2}{*}{} & \multicolumn{2}{|c|}{5 segments/elements } & \multicolumn{2}{c|}{10 segments/elements } & \multicolumn{2}{c|}{20 segments/elements } \\
\cline { 2 - 7 } & Present & Abaqus & Present & Abaqus & Present & Abaqus \\
\hline No. of load increments & 1 & 36 & 1 & 51 & 1 & 51 \\
\hline No. of iterations & 41 & 135 & 41 & 226 & 41 & 227 \\
\hline
\end{tabular}

Table 3: Predicted displacement components of point 3 under the point load, see Figure 11.

\begin{tabular}{|c|c|c|c|c|}
\hline & & $U[\mathrm{~m}]$ & $V[\mathrm{~m}]$ & $W[\mathrm{~m}]$ \\
\hline \multirow{3}{*}{ Present } & 5 segments & -4.805 & 0.000 & -34.984 \\
\hline & 10 segments & -4.803 & 0.000 & -34.965 \\
\hline & 20 segments & -4.802 & 0.000 & -34.960 \\
\hline \multirow{4}{*}{ Abaqus } & 5 elements & -4.848 & 0.000 & -35.461 \\
\hline & 10 elements & -4.803 & 0.000 & -34.967 \\
\hline & 20 elements & -4.802 & 0.000 & -34.962 \\
\hline & 100 elements & -4.802 & 0.000 & -34.961 \\
\hline \multicolumn{2}{|c|}{2 catenary elements [15] } & -4.786 & 0.000 & -34.902 \\
\hline \multicolumn{2}{|c|}{10 truss elements [15] } & -4.772 & 0.000 & -34.747 \\
\hline
\end{tabular}

\subsection{Static Analysis of a Pretensioned Cable Net}

This example was described by Tibert [17], among others, and also employed in the verification manual of SAP2000 [26]. The cable properties include $A=146.45 \mathrm{~mm}^{4}, E A=12.117 \mathrm{MN}$ and weight $=1.45939 \mathrm{~N} / \mathrm{m}$. The initial pretensioned state is set up as shown in Figure 12. The Z-axis defines the vertical upward direction. B to I are eight leveled anchor points. D-E and F-G are 
parallel to the $\mathrm{X}$-axis whilst $\mathrm{B}-\mathrm{H}$ and $\mathrm{C}-\mathrm{I}$ are parallel to the $\mathrm{Y}$-axis. The pretensions and the locations of the cable junctions 1 to 4 are adjusted such that junctions 1 to 4 are 9.144 m vertically below the intersecting points of D-E, F-G, B-H and C-I. On the other hand, the pretensions in 1-2, 3-4, 1-3 and 2-4 are adjusted to $24.2828 \mathrm{kN}$ and those in the more inclined portions (i.e., B-1, C-2, D-1, 2-E, F-3, 4-G, 3-H and 4-I) are adjusted to $23.6868 \mathrm{kN}$. Then, the forces holding the cable junctions are removed whilst a $35.586 \mathrm{kN}$ download force is applied to each of the freed cable junctions. The displacement components obtained from the RF cable element, Abaqus's B31 element, Tibert's catenary element and SAP2000's catenary element are listed in Table 4. All of them produce similar results which show that the present RB cable element is acceptable for the spatial cable net analysis.

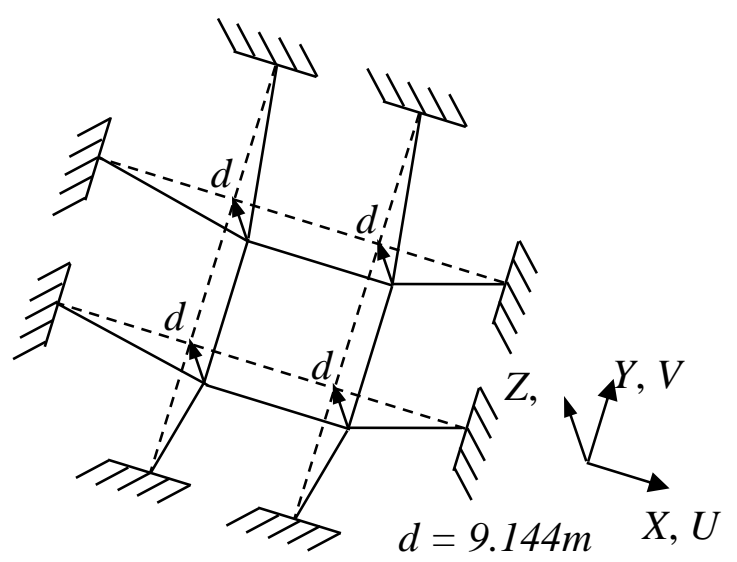

(a)

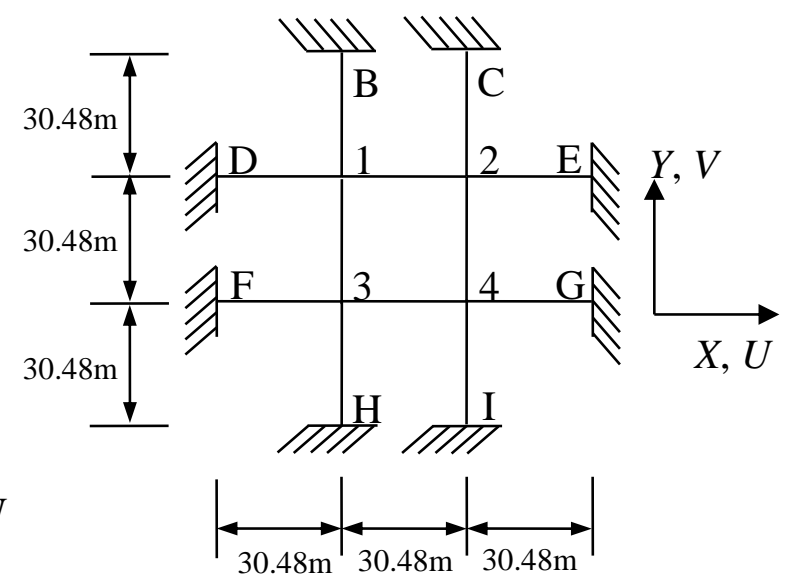

(b)

Figure 12. The initial configuration of a cable net: (a) isometric view, (b) top view.

Table 4. Predicted displacement components of joint 1, see Figure 12.

\begin{tabular}{|c|c|c|c|c|}
\hline \multicolumn{2}{|c|}{} & $U[\mathrm{~m}]$ & $V[\mathrm{~m}]$ & $W[\mathrm{~m}]$ \\
\hline \multirow{2}{*}{ Present } & 24 segments & -0.04033 & 0.04033 & -0.44773 \\
& 48 segments & -0.04033 & 0.04033 & -0.44773 \\
\hline Abaqus & 24 elements & -0.04059 & 0.04059 & -0.45075 \\
& 48 elements & -0.04060 & 0.04060 & -0.45082 \\
\hline \multicolumn{2}{|c|}{12 catenary elements of Tibert [17] } & -0.04048 & 0.04048 & -0.45001 \\
\hline \multicolumn{2}{|c|}{12 catenary elements of SAP2000 [26] } & -0.04029 & 0.04029 & -0.44766 \\
\hline
\end{tabular}

\subsection{Static Analysis of Cable}

The cable in this example was considered by Jayarman \& Knudson [15]. With the unstretched length equal to $253.746 \mathrm{~m}$, the cable is anchored at two leveled points at separation of $254 \mathrm{~m}$ as shown in Figure 13. Properties of the cable include: $A=41.93 \mathrm{~mm}^{4}, E A=5.7821 \mathrm{MN}$ and weight $=$ $3.5 \mathrm{~N} / \mathrm{m}$. The upward direction is defined by the Z-axis. Same as Jayarman \& Knudson [15], only 
half of the cable is modeled due to symmetry. Again, the weight over an element is lumped as the nodal force acting on the central node of the element. Table 5 lists the amount of sag, which is the vertical distance between the anchoring points and the midpoint $B$ of the cable, predicted by the present formulation, Abaqus and a catenary element. The predictions do not differ from each other by more than $1.5 \%$.

Table 5. Static sag $[\mathrm{m}]$ of the cable in Figure 13.

\begin{tabular}{|c|c|c|c|c|c|c|}
\hline \multicolumn{3}{|c|}{ Present } & \multicolumn{3}{c|}{ Abaqus B31 beam element } & \multirow{2}{*}{$\begin{array}{c}\text { catenary } \\
\text { element [15] }\end{array}$} \\
\cline { 1 - 6 } 2 segments & 5 segments & 10 segments & 2 elements & 5 elements & 10 elements & (15.343 \\
\hline 3.374 & 3.339 & 3.334 & 3.383 & 3.348 & 3.334 & 3.348 \\
\hline
\end{tabular}

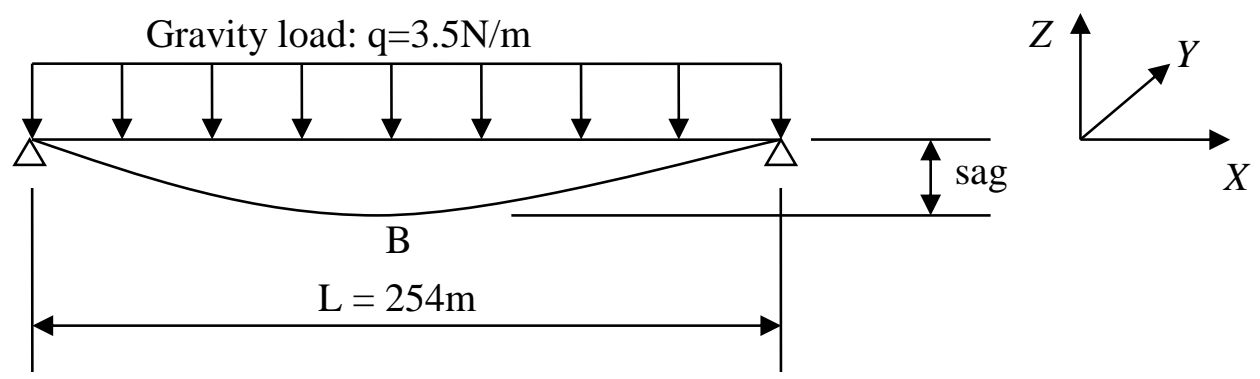

Figure 13. Static equilibrium of a cable under its own weight.

\subsection{Dynamic Analysis of Cable}

In this subsection, the dynamic response of the cable in Figure 13 is studied. With the cable assuming its static equilibrium configuration, a uniform downward load of $35 \mathrm{~N} / \mathrm{m}$, which is 10 times of the cable weight, is applied instantaneously. Same as the distributed load, the mass of an element is lumped to its central node and the arrangement is suitable for explicit time integration. The vertical displacement of point $B$ is computed by modeling half of the cable with different numbers of RF segments and B31 elements. The highest eigen frequencies of these assemblages and the critical time steps estimated by $1 / \pi f$ for stable predictions are given in Table 6 . In particular, the 10-segment and 5-element assemblages possess 33 and 36 dofs, respectively, and their critical time steps are also close. It should be remarked that the estimated critical time step $1 / \pi f$ only works for linear analysis and may not work for the entire time history for nonlinear problems. To secure stable predictions for the 10-segment and 5-element assemblages, the related time steps are both taken as $1 \mathrm{~ms}$ which is about one-third of their $1 / \pi f$. Their predictions for the vertical deflection of B are plotted in Figure 14(a). The prediction of the 20-element assemblage is taken as the reference solution and more elements do not lead to any practical change in the result. It can be seen that the 
result produced by 10 -segment assemblage is far closer to the reference solution than that of the element assemblage. The prediction of the 20-segment assemblage is included to check using whether more segments would improve the prediction and the result is positive.

Table 6. Highest eigen frequencies of the assemblages modeling the cable problem in Figure 13.

\begin{tabular}{|c|c|c|c|c|c|}
\hline \multirow{2}{*}{} & \multicolumn{2}{|c|}{ No. of RF segments } & \multicolumn{3}{|c|}{ No. of B31 beam elements } \\
\cline { 2 - 6 } & 10 & 20 & 5 & 10 & 20 \\
\hline Highest eigen frequency $f[$ cycle/s] & 99.8 & 201.5 & 109.3 & 218.1 & 437.0 \\
\hline $1 / \pi f[\mathrm{~ms}]$ & 3.19 & 1.56 & 2.91 & 1.46 & 0.73 \\
\hline
\end{tabular}

With the cable under static equilibrium again, the Y-directional distributed impulsive load portrayed in Figure 14(b) with 0.2 second duration and $35 \mathrm{~N} / \mathrm{m}$ peak is applied to the cable. The $\mathrm{Y}$ and Z- displacements of point B predicted by the same set of assemblies are shown in Figures 14(c) and 14(d), respectively. It can be seen that the result produced by the 10-segment assemblage is also closer to the reference solution, produced by the 20-element assemblage, than the 5-element assemblage.

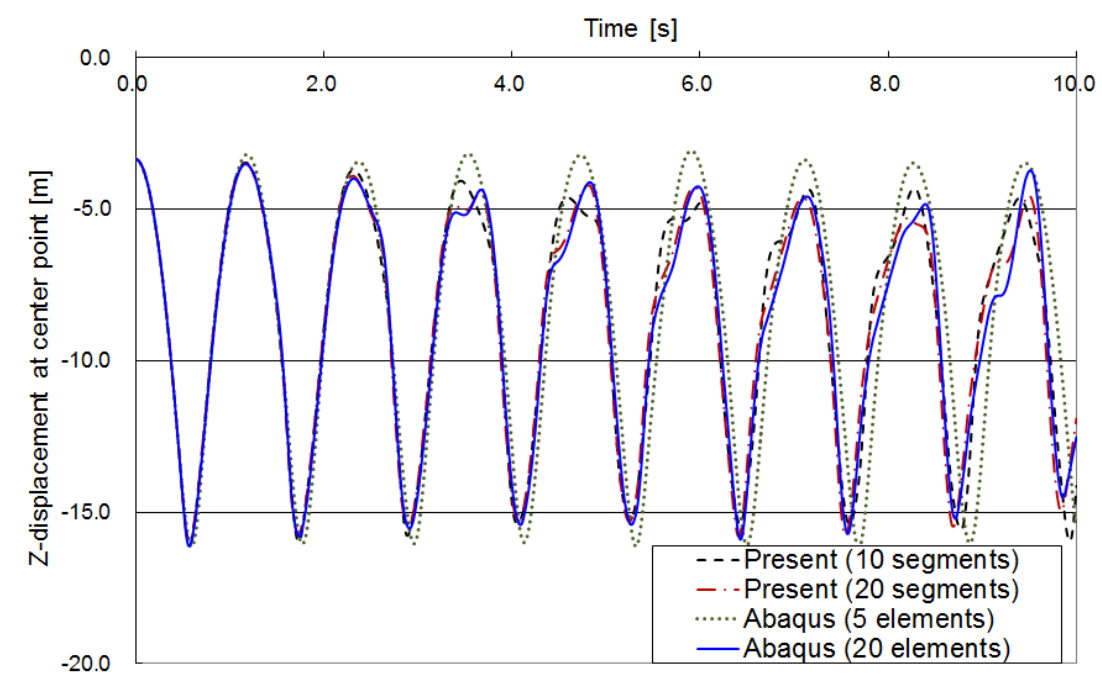

(a) 


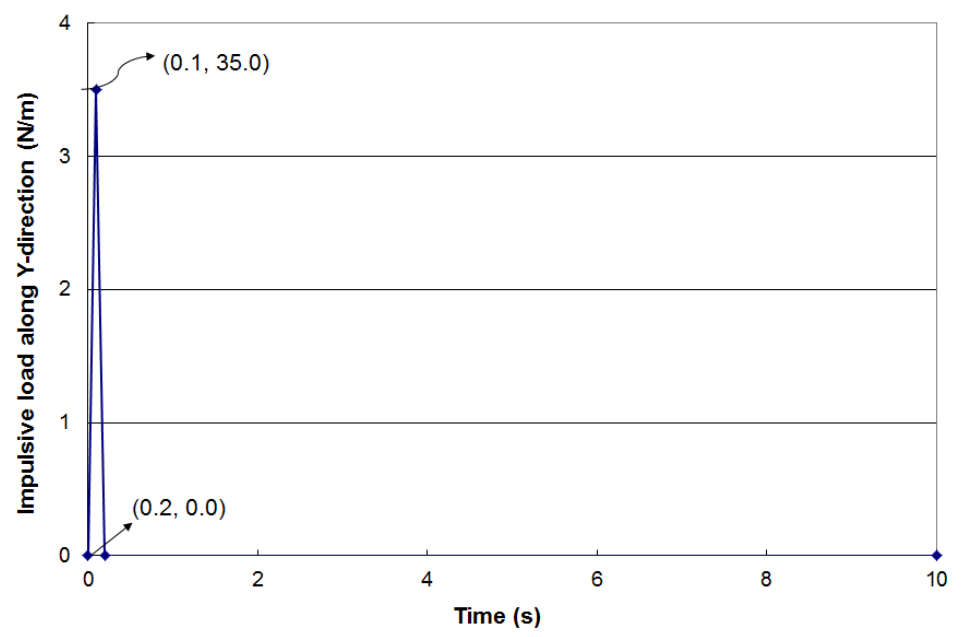

(b)

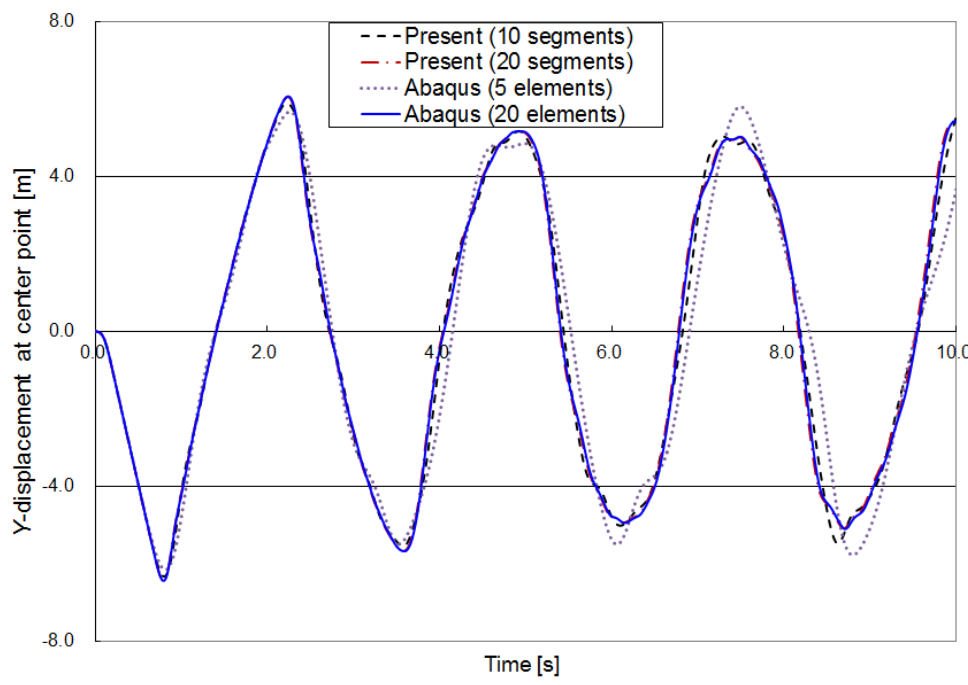

(c)

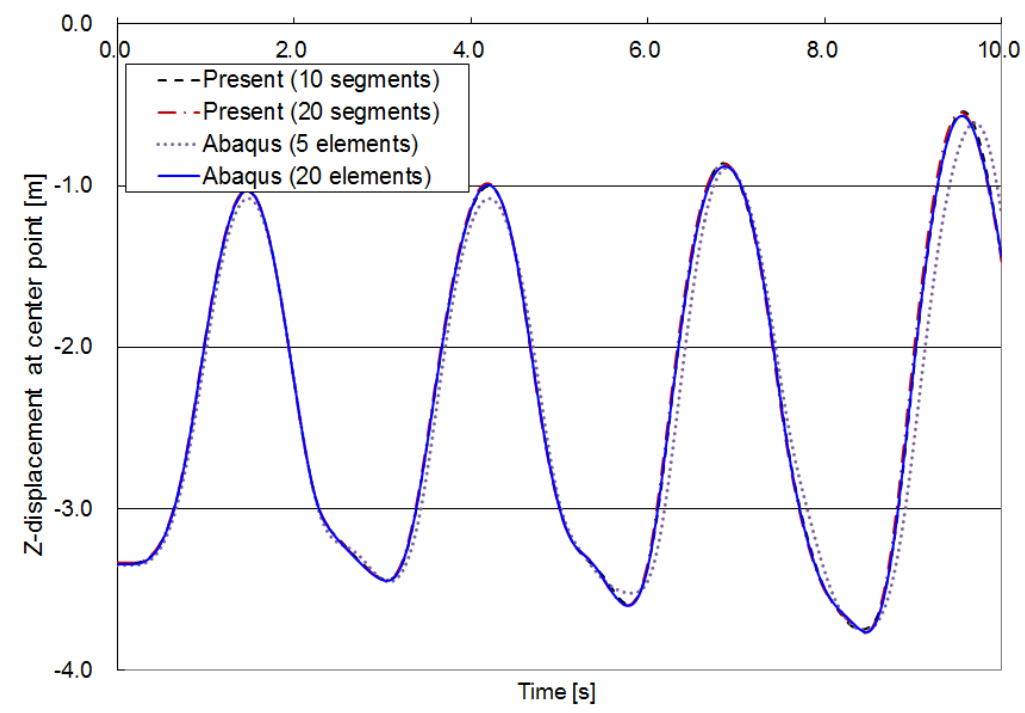

(d)

Figure 14. (a) Z-displacement of point $B$ after a $35 \mathrm{~N} / \mathrm{m}$ instantaneous load along the Z-direction is applied to the cable in Figure 13. (b) The impulsive load along Y-direction. (c) Y-displacement of point B after the impulsive load in (b). (d) Z-displacement of point B after the impulsive load in (b). 


\subsection{Cable subjected to periodic ground motion}

Figure 15 shows a cable with unstretched length $10 \mathrm{~m}$ anchored at two leveled points $10 \mathrm{~m}$ apart. The two points are subjected to the same periodic X-displacement $\sin \omega t$ where $\omega=1 \mathrm{~s}^{-1}$ and $\omega=10 \mathrm{~s}^{-1}$ are attempted. A lumped mass $\mathrm{M}=100 \mathrm{~kg}$ is attached to the midpoint of the cable. The cable properties as well as the treatments for the distributed gravity load and the mass are the same as those in the last example. The cable is modeled by 12 and 40 RF-segments as well as 6 and 40 B31 elements. The highest eigen frequencies and the estimated critical time steps are given in Table 7. Similar to the last subsection, the time step for the 6-element assemblages are taken to be $0.06 \mathrm{~ms}$ which again is approximately one-third of the estimated critical time steps. However, Abaqus fails with the warning message "excessively distorted element" returned. The warning message disappears when the time step is reduced to $0.0091 \mathrm{~ms}$. The purpose of the 40-element assembly is to obtain a reference solution. However, the warning message "excessively distorted element" keeps appearing even when the time step is taken to be $0.00001 \mathrm{~ms}$. Further reducing the time step leads to "system error". On the other hand, both the 12 and 40 RF-segments are able to produce predictions with the time steps slightly lower than the estimated critical time steps.

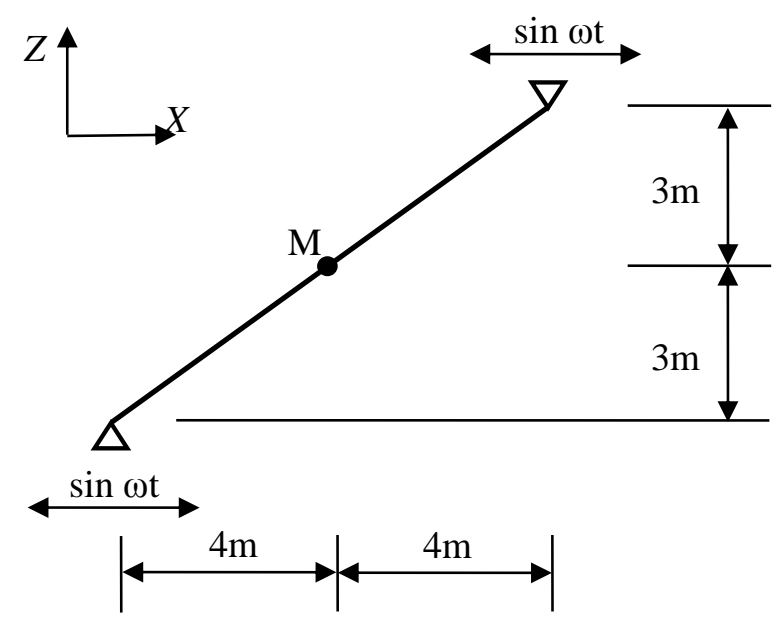

Figure 15. A cable with a concentrated mass $M=100 \mathrm{~kg}$ attached to its midpoint. X-displacement of the concentrated mass at two driving frequencies $\omega=1 \mathrm{~s}^{-1}$ and $\omega=10 \mathrm{~s}^{-1}$ is predicted.

Table 7. Highest eigen frequencies of the assemblages modeling the cable problem in Figure 15.

\begin{tabular}{|c|c|c|c|c|}
\hline \multirow{2}{*}{} & \multicolumn{2}{|c|}{ No. of RF segments } & \multicolumn{2}{c|}{ No. of B31 beam elements } \\
\cline { 2 - 5 } & 12 & 40 & 6 & 40 \\
\hline Highest eigen frequency $f[\mathrm{cycle} / \mathrm{s}]$ & 1486 & 5113 & 1664 & 11086 \\
\hline $1 / \pi f[\mathrm{~ms}]$ & 0.214 & 0.062 & 0.191 & 0.029 \\
\hline
\end{tabular}

It is conjectured that the warning message may be caused by finite rotation which is a non-trivial 
issue in three-dimensional problems. As the cable motion should be confined in the X-Z-plane, the Y-displacement, X-rotation and Z-rotation of all the beam elements are restrained. After applying the constraints, the problem becomes planar. The restrained beam assemblages are able to produce prediction with the time steps as guarded by the estimated critical time steps which remains unchanged.

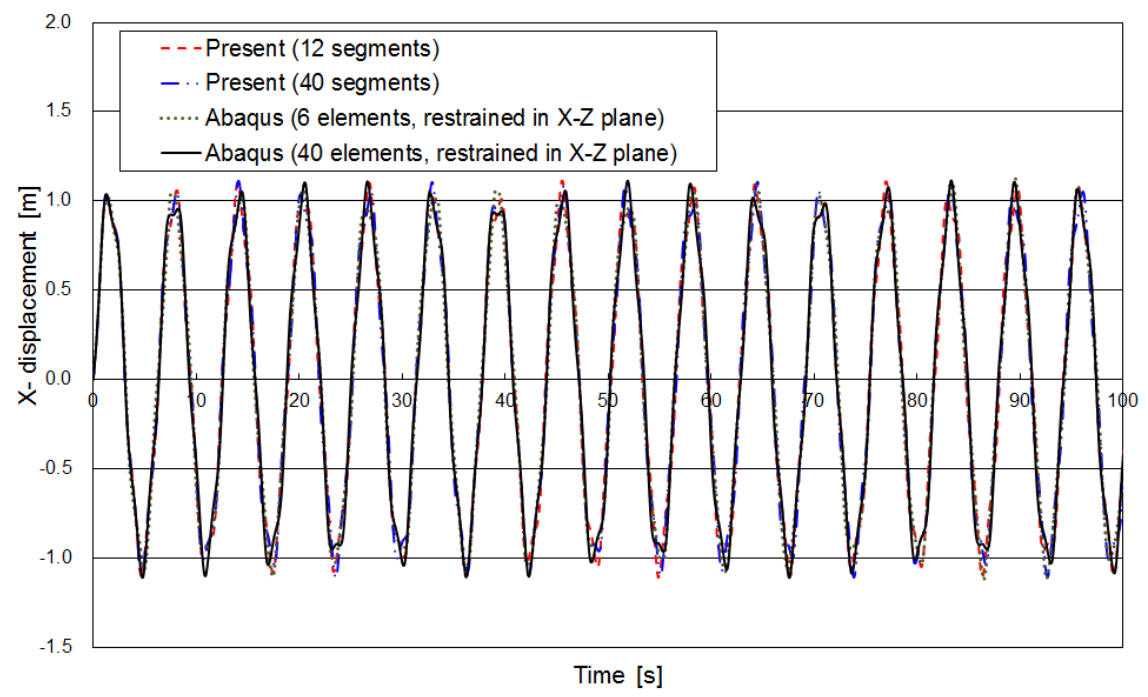

(a)

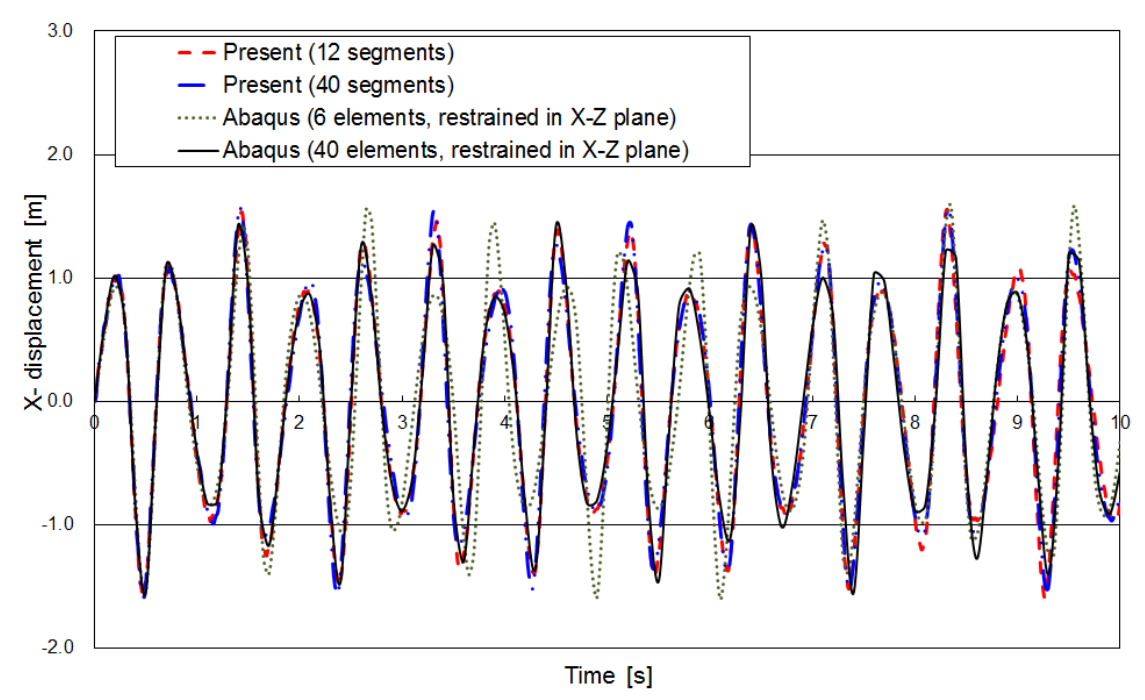

(b)

Figure 16. The predicted $\mathrm{X}$-displacement of the $100 \mathrm{~kg}$ mass in Figure 15 for (a) $\omega=1 \mathrm{~s}^{-1}$ and (b) $\omega=10 \mathrm{~s}^{-1}$.

Figure 16(a) and 16(b) show the X-displacement of the concentrated mass for $\omega=1$ and $\omega=10$ $\mathrm{s}^{-1}$, respectively. The results are predicted by the 12 and $40 \mathrm{RF}$-segments as well as 6 and 40 restrained B31 elements. The time steps used by the 12-segement and 6-element assemblages are both taken to be $0.06 \mathrm{~ms}$. When $\omega=1 \mathrm{~s}^{-1}$, all predictions are close. When $\omega=10 \mathrm{~s}^{-1}$, the result 
produced by the 12-segment assemblage is clearly closer to the reference solution, produced by the 40-element assemblage, than the 6-element assemblage.

\section{Closure}

A simple rotation-free beam element formulation previously developed by the authors is re-visited and extended to a pseudo 3D formulation for the spatial cable analyses. Comparing with other rotation-free beam elements, the present one is probably the simplest but its accuracy is competitive. By using a corotational approach, a geometrically nonlinear formulation can be achieved. For straight beams, the bending energy can be expressed as a quadratic function of the nodal displacements and the tangent bending stiffness matrix is a constant matrix. For curved beams, the tangent bending stiffness matrix can also be approximated as a constant matrix with an insignificant adverse effect on the convergence. Numerical examples attempted by other rotation-free elements are first examined here. The results show that the present formulation can give satisfactory predictions in both linear and geometrically nonlinear analyses. The superior features of the rotation-free beam formulation also make it a promising choice for cable analyses. To this end, the plane beam formulation is extended to a pseudo 3D beam formulation in which the torsional stiffness can be neglected and the sectional properties are isotropic. A number of examples on cable analyses are examined and the yielded predictions are close to those of the thin beam finite elements. However, the tolerable load increment and maximum stable time step of the rotation-free element far exceed those of the two-node thin-beam finite element model in nonlinear static and explicit dynamic analyses, respectively.

It should be mentioned that the major advantage of the present rotation-free beam element is its efficiency in terms of the large tolerable load increment, large stable time step and absence of the finite rotational problem. Its plate/shell counterpart has been applied to fabric drape simulation in which the finite element method has rarely gained any success [11,27]. Other rotation-free plate/shell elements have been applied to metal forming problems and drap simulation [6,7]. The simplicity and efficiency of the present rotation-free framework are restricted to small strain problem. Inclusion of large strains needs to be further explored.

Acknowledgment - This work is supported by the Hong Kong Research Grant Council in the form of a GRF grant (HKU 7173 09E). 


\section{References}

1] R. Phaal, C.R. Calladine, A simple class of finite elements for plate and shell problems. I: Elements for beams and thin flat plates, Int. J. Numer. Meth. Eng., 35 (1992) 955-977.

[2] R. Phaal, C.R. Calladine, A simple class of finite elements for plate and shell problems. II: an element for thin shells, with only translational degrees of freedom, Int. J. Numer. Meth. Eng., 35 (1992) 979-996.

[3] E. Oñate, F. Zarate, Rotation-free triangular plate and shell elements, Int. J. Numer. Meth. Eng., 47 (2000) 557-603.

[4] F.G. Flores, E. Oñate, Improvements in the membrane behaviour of the three node rotation-free BST shell triangle using an assumed strain approach, Comput. Meth. Appl. Mech. Eng., 194 (2005) 907-932.

[5] F. Sabourin, M. Brunet, Detailed formulation of the rotation-free triangular element "S3" for general purpose shell analysis, Eng. Computation., 23 (2006) 469-502.

[6] Y. Guo, W. Gati, H. Naceur, J. Batoz, An efficient DKT rotation free shell element for springback simulation in sheet metal forming, Comput. Struct., 80 (2002) 2299-2312.

[7] F.G. Flores, E. Oñate, Wrinkling and folding analysis of elastic membranes using an enhanced rotation-free thin shell triangular element, Finite Elem. Anal. Des., 47 (2011) 982-990.

[8] F.G. Flores, E. Oñate, Rotation-free finite element for the non-linear analysis of beams and axisymmetric shells, Comput. Meth. Appl. Mech. Eng., 195 (2006) 5297-5315.

[9] E. Oñate, F. Zarate, Extended rotation-free plate and beam elements with shear deformation effects, Int. J. Numer. Meth. Eng., 83 (2010) 196-227.

[10] J.M. Battini, A rotation-free corotational plane beam element for non-linear analyses, Int. J. Numer. Meth. Eng., 75 (2008) 672-689.

[11] Y.X. Zhou, K.Y. Sze, A Geometric Nonlinear Rotation-free Triangle \& its application in Drape Simulation, Int. J. Numer. Meth. Eng., in press (2011).

[12] H.T. Thai, S.E. Kim, Nonlinear static and dynamic analysis of cable structures, Finite Elem. Anal. Des., 47 (2011) 237-246.

[13] H. Ozdermir, A finite element approach for cable problems, Int. J. Solids. Struct., 15 (1979) 429-437.

[14] Z.H. Chen, Y.J. Wu, Y. Yin, C. Shan, Formulation and application of multi-node sliding cable element for the analysis of suspen-dome structures, Finite Elem. Anal. Des., 46 (2010) 743-750.

[15] H.B. Jayaraman, W.C. Knudson, A curved element for the analysis of cable structures, Comput. Struct., 14 (1981) 325-333.

[16] A. Andreu, L. Gil, P. Roca, A new deformable catenary element for the analysis of cable net structures, Comput. Struct., 84 (2006) 1882-1890.

[17] G. Tibert, Numerical analyses of cable roof structures, Licentiate Thesis, Royal Institute of Technology, Stockholm, Sweden, 1999.

[18] C.J. Wang, R.P. Wang, S.L. Dong, R.J. Qian, A new catenary cable element, Int. J. Space. Struct., 18 (2003) 269-275.

[19] H. Irvin, Cable structures, MIT PRESS, Cambridge, Mass, 1981.

[20] B. Buckham, F.R. Driscoll, M. Nahon, Development of a finite element cable model for use in low-tension dynamics simulation, J. Appl. Mech. ASME, 71 (2004) 476-485.

[21] H.M. Ali, A.M. Abdel-Ghaffar, Modeling the nonlinear seismic behavior of cable-stayed bridges with passive control bearings, Comput. Struct., 54 (1995) 461-492.

[22] A.A. Shabana, H.A. Hussien, J.L. Escanola, Application of the absolute nodal coordinate formulation to large rotation and large deformation problems, J. Mech. Design, 120 (1998) 188-195. [23] J.H. Seo, I.H. Jung, T.W. Park, J.B. Chai, Dynamic analysis of a multibody system including a 
very flexible beam element, JSME Int J. Ser. C, 48 (2005) 224-233.

[24] K.Y. Sze, X.H. Liu, A corotational interpolatory model for fabric drape simulation, Int. J. Numer. Meth. Eng., 77 (2009) 799-823.

[25] K.O. Papaliliou, Bending of helically twisted cables under variable bending stiffness due to internal friction, tensile force and cable curvature, Doctor of technical sciences Thesis, ETH, Athens, 1995.

[26] Computers \& Structures, Inc., SAP2000 Software Verification Manuals, Cable, Problems 7 003, http://www.comp-engineering.com/SAPManE.htm.

[27] K.Y. Sze, X.H. Liu, Fabric drape simulation by solid-shell finite element method. Finite Elem. Anal. Des., 43 (2007): 819-838. 